\title{
Efficient Asymmetric Synthesis of Quaternary $(E)$-Vinylglycines by Deconjugative Alkylation of Dehydroamino Acids
}

\author{
M. C. Jones, S. P. Marsden, ${ }^{\star}$ D. M. Muñoz-Subtil
}

\section{Supporting Information}

\section{Benzyloxycarbonylamino(diethoxyphosphoryl)acetic acid (-)-8-phenylmenthyl ester 8}<smiles>[CH][Po+]C(C(=O)O)[N+](=O)[O-]</smiles>

$\mathrm{NHCbz}$<smiles>CCOC(=O)NC(C(=O)OCC)C(=O)OCc1ccccc1</smiles>

8
Aux $=$<smiles>C[C@H]1CC[C@@H](C(C)(C)c2ccccc2)[C@H](C)C1</smiles>

EDC (20.4 g, $70.9 \mathrm{mmol})$ was added to a stirred solution of $\mathrm{N}$-Cbz-diethylphosphonoglycine ${ }^{1}(24.5 \mathrm{~g}, 70.9 \mathrm{mmol})$, (-)-8-phenylmenthol $(17.8 \mathrm{~g}, 76.8 \mathrm{mmol})$ and DMAP $(1.7 \mathrm{~g}, 14 \mathrm{mmol})$ in DCM $(100 \mathrm{ml})$ at room temperature under nitrogen. The reaction mixture was stirred for 4 days. The reaction mixture was diluted with DCM (200 ml) and washed successively with a $1 \mathrm{M}$ aqueous hydrogen chloride solution $(100 \mathrm{ml})$, a saturated aqueous sodium hydrogen carbonate solution $(100 \mathrm{ml})$ and brine $(50 \mathrm{ml})$. The organic extracts were dried $\left(\mathrm{Na}_{2} \mathrm{SO}_{4}\right)$, filtered and evaporated under reduced pressure to give a crude product, which was purified by flash chromatography, eluting with 1:1 petrol-ethyl acetate, to give recovered (-)-8-phenylmenthol $(4.88 \mathrm{~g})$ as a colourless oil and phosphonate 8 $\left(27.76 \mathrm{~g}, 70 \%, 88: 12\right.$ mixture of diastereoisomers) as a colourless oil; $R_{\mathrm{F}} 0.4\left(50 \%\right.$ EtOAc in petrol); $[\alpha]_{\mathrm{D}}{ }^{24}+20.68$ (c. 9.6 in $\mathrm{CHCl}_{3}$ ); (Found: $\mathrm{C}, 64.1 ; \mathrm{H}, 7.75 ; \mathrm{N}, 2.60 ; \mathrm{P}, 5.45 ; \mathrm{C}_{30} \mathrm{H}_{42} \mathrm{NPO}_{7}$ requires: $\mathrm{C}, 64.39 ; \mathrm{H}, 7.56 ; \mathrm{N}, 2.50 ; \mathrm{P}$, $5.53 \%) ; v_{\max } / \mathrm{cm}^{-1}\left(\mathrm{CHCl}_{3}\right.$ solution) $3246,1725,1536,1497,1306,1266,1213,1047$ and 1027; $\delta_{\mathrm{H}}(300 \mathrm{MHz}$, $\left.\mathrm{CDCl}_{3}\right)$ 7.45-7.05 (10H, m, Ph), $5.31\left(1 \mathrm{H}, \mathrm{brd}, J\right.$ 8.5, NH), $5.15\left(2 \mathrm{H}, \mathrm{s}, \mathrm{CH}_{2} \mathrm{Ph}(\mathrm{Cbz})\right), 4.89(1 \mathrm{H}, \mathrm{td}, J 10.8$ and 4.1, $\mathrm{CHOCO})$, 3.85-4.23 (5H, m, $\mathrm{OCH}_{2} \mathrm{CH}_{3} \times 2$ and $\left.\mathrm{P}-\mathrm{CH}\right)$ and 2.10-0.80 $(23 \mathrm{H}, \mathrm{m}$, aliphatic $\mathrm{H})$; peak visible for minor isomer at $4.68\left(1 \mathrm{H}, \mathrm{dd},{ }^{2} \mathrm{~J}_{\mathrm{P}-\mathrm{H}} 22.0\right.$ and J 9.2, $\left.\mathrm{P}-\mathrm{CH}\right)$; $\delta_{\mathrm{H}}\left(500 \mathrm{MHz}, \mathrm{d}_{6}\right.$-DMSO, 300K) $7.96(1 \mathrm{H}, \mathrm{brd}, J 8.9, \mathrm{NH}), 7.43-$ $7.07(10 \mathrm{H}, \mathrm{m}, \mathrm{Ph}), 5.13\left(1 \mathrm{H}, \mathrm{d}, J 12.5, \mathrm{CH}_{A} \mathrm{H}_{\mathrm{B}} \mathrm{Ph}(\mathrm{Cbz})\right), 5.09\left(1 \mathrm{H}, \mathrm{d}, J 12.5, \mathrm{CH}_{\mathrm{A}} \mathrm{H}_{B} \mathrm{Ph}(\mathrm{Cbz})\right), 4.71(1 \mathrm{H}, \mathrm{td}, J 10.6$ and 4.2, CHOCO), $4.19\left(1 \mathrm{H}, \mathrm{dd},{ }^{2} J_{\mathrm{P}-\mathrm{H}} 23.5\right.$ and J 9.2, P-CH), $4.07\left(1 \mathrm{H}\right.$, apparent q, J 7.7, $\left.\mathrm{OCH}_{A} \mathrm{H}_{\mathrm{B}} \mathrm{CH}_{3}\right)$, 3.98-3.80 $\left(3 \mathrm{H}, \mathrm{m}, \mathrm{OCH}_{\mathrm{A}} \mathrm{H}_{\mathrm{B}} \mathrm{CH}_{3}\right.$ and $\left.\mathrm{OCH}_{2} \mathrm{CH}_{3}\right)$ and 2.02-0.77 $(23 \mathrm{H}, \mathrm{m}$, aliphatic $\mathrm{H})$; peaks visible for minor isomer at $8.16(1 \mathrm{H}$, brd, J 8.6, NH), $5.04\left(1 \mathrm{H}, \mathrm{d}, J 12.5, \mathrm{CH}_{\mathrm{A}} H_{B} \mathrm{Ph}(\mathrm{Cbz})\right)$, and $4.59\left(1 \mathrm{H}, \mathrm{dd},{ }^{2} J_{\mathrm{P}-\mathrm{H}} 23.3\right.$ and J 9.4, $\left.\mathrm{P}-\mathrm{CH}\right) ; \delta_{\mathrm{C}}(75 \mathrm{MHz}$, $\left.\mathrm{CDCl}_{3}\right) 166.3,155.6\left({ }^{3} J_{P-C} 5.7\right), 151.8,136.5,128.9,128.7,128.6,128.4,126.0,125.9,77.3,67.7,63.9\left({ }^{2} J_{P-C} 6.8\right)$, $63.7\left({ }^{2} J_{\text {P-C }} 6.7\right.$ ), $52.7\left({ }^{1} J_{P-C} 146.9\right), 51.0,41.3,39.9,34.8,31.7,29.2,26.8,24.2,22.2,16.7$ and 16.7; peaks visible for minor isomer at 165.6, 150.8, 136.3, 128.8, 128.1, 78.2, 67.9, $\left.64.1{ }^{2}{ }^{2} J_{P-C} 9.1\right), 53.7\left({ }^{1} J_{P-C} 148.0\right), 50.7,41.3$, 39.9, 34.7, 31.8, 28.8, 27.5, and 25.3; $\delta_{\mathrm{P}}\left({ }^{31} \mathrm{P}, 202 \mathrm{MHz}, \mathrm{d}_{6}\right.$-DMSO) 17.80 and 17.48; $\mathrm{m} / \mathrm{z}$ (low resolution, ES) 560.1 $\left(100 \%, M^{+}+H\right)$.

\footnotetext{
${ }^{1}$ R. Shankar and A. I. Scott, Tetrahedron Lett. 1993, 34, 231-234.
} 


\section{General procedure for formation of dehydroamino acid esters 5}

DBU or tetramethylguanidine (1.0 equivalent) was added to a stirred solution of phosphonate 8 (1.0 equivalent) in DCM (0.5 M, typical concentration) at room temperature under nitrogen. The reaction mixture was stirred for 30 minutes. The aldehyde was then added (as a solution in DCM when required) and stirring continued for 24 hours. The reaction mixture was absorbed directly onto silica and purified by flash chromatography, typically eluting with petrol-diethyl ether, to give the (Z)-dehydroamino acid esters $\mathbf{5}$.

\section{(Z)-2-Benzyloxcarbonylaminododec-2-enoic acid (-)-8-phenylmenthyl ester 5a}<smiles>O=C(NC(=CCC[18OH])C(=O)O[Na])OCc1ccccc1</smiles>

Using tetramethylguanidine. Colourless oil; $R_{\mathrm{F}} 0.15$ (5\% EtOAc in petrol); $[\alpha]_{\mathrm{D}}^{23}+31.8$ (c. 4.65 in $\mathrm{CHCl}_{3}$ ); (Found: C, 76.9; $\mathrm{H}, 9.3 ; \mathrm{N}, 2.4 ; \mathrm{C}_{36} \mathrm{H}_{51} \mathrm{NO}_{4}$ requires: $\left.\mathrm{C}, 76.97 ; \mathrm{H}, 9.15 ; \mathrm{N}, 2.49 \%\right) ; v_{\max } / \mathrm{cm}^{-1}\left(\mathrm{CHCl}_{3}\right.$ solution) 3390, 1716, 1658, 1497, 1455, 1257 and 1219; $\delta_{\mathrm{H}}\left(300 \mathrm{MHz} \mathrm{CDCl}_{3}\right)$ 7.45-7.05 $(10 \mathrm{H}, \mathrm{m}, \mathrm{Ph}), 6.24(1 \mathrm{H}, \mathrm{t}, J 7.4, \mathrm{H}-3), 5.12(1 \mathrm{H}$, d, J 12.5, $\left.\mathrm{CH}_{A} \mathrm{H}_{\mathrm{B}} \mathrm{Ph}(\mathrm{Cbz})\right), 5.03\left(1 \mathrm{H}, \mathrm{d}, J 12.5, \mathrm{CH}_{\mathrm{A}} \mathrm{H}_{B} \mathrm{Ph}(\mathrm{Cbz})\right), 5.18-4.98(1 \mathrm{H}, \mathrm{brs}, \mathrm{NH}), 4.87(1 \mathrm{H}, \mathrm{td}, J 10.8$ and 4.4, $\mathrm{CHOCO})$ and 2.18-0.83 $(36 \mathrm{H}, \mathrm{m}$, aliphatic $\mathrm{H}) ; \delta_{\mathrm{C}}\left(75 \mathrm{MHz}, \mathrm{CDCl}_{3}\right) 163.8,154.4,152.7,139.8,136.8,128.9$, 128.5, 128.5, 128.5, 128.4, 125.8, 125.3, 75.6, 67.3, 50.7, 41.8, 39.8, 34.9, 32.3, 31.7, 29.9, 29.8, 29.8, 29.7, 29.7, 28.7, 28.5, 26.8, 23.7, 23.1, 22.2 and 14.6; m/z (low resolution, ES) $579.5\left(31 \%, \mathrm{M}^{+}+\mathrm{NH}_{4}{ }^{+}\right)$and $562.5(100 \%$, $\left.\mathrm{M}^{+}+\mathrm{H}\right)$.

(Z)-2-Benzyloxycarbonylamino-4-phenylbut-2-enoic acid (-)-8-phenylmenthyl ester 5b

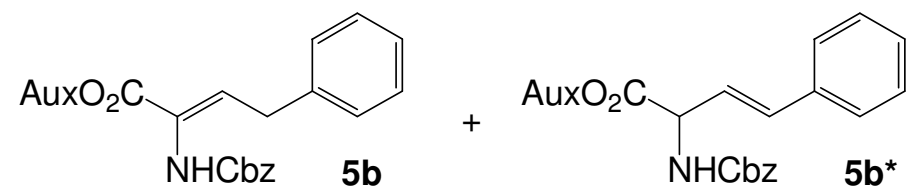

Using DBU. Colourless oil ( ${ }^{1} \mathrm{H}-\mathrm{NMR}$ analysis revealed the product to be a 7:3 mixture of the dehydroamino ester $\mathbf{5 b}$ and its deconjugated isomer $5 \mathbf{b}^{*}$ as a $3: 1$ mixture of diastereoisomers); $R_{\mathrm{F}} 0.26$ (20\% $\mathrm{Et}_{2} \mathrm{O}$ in petrol); $[\alpha]_{\mathrm{D}}{ }^{25}+5.3$ (c. 6.5 in $\left.\mathrm{CHCl}_{3}\right) ; v_{\max } / \mathrm{cm}^{-1}\left(\mathrm{CHCl}_{3}\right.$ solution) $3383,1715,1657,1600,1496,1455,1296,1256,1221 ; \delta_{\mathrm{H}}(300 \mathrm{MHz}$, $\left.\mathrm{CDCl}_{3}\right)$ 7.5-7.2 (15H, m, Ph), $6.36\left(1 \mathrm{H}, \mathrm{t}, J\right.$ 7.4, H-3), $5.14\left(1 \mathrm{H}, \mathrm{d}, J 12.3, \mathrm{CH}_{A} \mathrm{H}_{\mathrm{B}} \mathrm{Ph}(\mathrm{Cbz})\right), 5.07(1 \mathrm{H}, \mathrm{d}, J 12.7$, $\left.\mathrm{CH}_{\mathrm{A}} H_{B} \mathrm{Ph}(\mathrm{Cbz})\right), 4.79(1 \mathrm{H}, \mathrm{td}, J 10.8$ and 4.3, CHOCO), $3.38(2 \mathrm{H}, \mathrm{d}, J 7.7, \mathrm{H}-4)$ and 2.15-0.79 (18H, m, aliphatic $\mathrm{H})$; signals visible for minor isomers at $6.63(1 \mathrm{H}, \mathrm{d}, J 16.7, \mathrm{H}-4), 6.12(1 \mathrm{H}, \mathrm{dd}, J 16.2$ and 7.0, $\mathrm{H}-3), 5.87(1 \mathrm{H}, \mathrm{dd}, J$ 15.4 and $4.8, \mathrm{H}-3)$ and $4.27(1 \mathrm{H}$, apparent $\mathrm{t}, J$ 7.2, $\mathrm{H}-2) ; \delta_{\mathrm{C}}\left(75 \mathrm{MHz}, \mathrm{CDCl}_{3}\right)$ 163.2, 152.2, 136.3, 128.7, 128.6, 128.5, 128.2, 128.1, 128.0, 127.9, 126.6, 126.6, 126.4, 125.6, 125.3, 124.9, 75.4, 67.1, 50.3, 41.4, 39.4, 34.5, 34.5, 31.3, 29.4, 26.3, 23.1 and 21.7; $\mathrm{m} / \mathrm{z}$ (low resolution, ES) $526.0\left(100 \%, \mathrm{M}^{+}+\mathrm{H}\right) ; \mathrm{m} / \mathrm{z}$ (accurate mass, ES) Found: $\mathrm{M}^{+}+{ }^{23} \mathrm{Na}, 548.2772 . \mathrm{C}_{34} \mathrm{H}_{39} \mathrm{NO}_{4} \mathrm{Na}$ requires $\mathrm{M}, 548.2777$. 


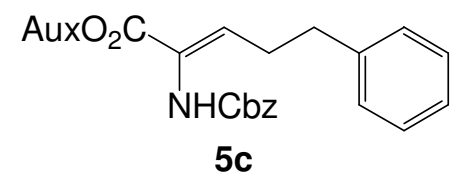

Using DBU. Colourless oil; $R_{\mathrm{F}} 0.23\left(15 \% \mathrm{Et}_{2} \mathrm{O}\right.$ in petrol); $[\alpha]_{\mathrm{D}}^{25}+2.4$ (c. $\left.9.5 \mathrm{in} \mathrm{CHCl}_{3}\right) ; v_{\max } / \mathrm{cm}^{-1}\left(\mathrm{CHCl}_{3}\right.$ solution) 3386, 3323, 1738, 1714, 1659, 1601, 1496, 1454, 1265 and 1221; $\delta_{\mathrm{H}}\left(300 \mathrm{MHz}, \mathrm{CDCl}_{3}\right) 7.01-7.50(15 \mathrm{H}, \mathrm{m}, \mathrm{Ph})$, $6.22(1 \mathrm{H}, \mathrm{t}, J 7.4, \mathrm{H}-3), 5.11\left(1 \mathrm{H}, \mathrm{d}, J 12.4, \mathrm{CH}_{A} \mathrm{H}_{\mathrm{B}} \mathrm{Ph}(\mathrm{Cbz})\right), 5.03\left(1 \mathrm{H}, \mathrm{d}, J 12.4, \mathrm{CH}_{\mathrm{A}} \mathrm{H}_{B} \mathrm{Ph}(\mathrm{Cbz})\right), 4.86(1 \mathrm{H}, \mathrm{td}, J$ 10.8 and 4.3, CHOCO), $2.68(2 \mathrm{H}, \mathrm{m}, \mathrm{H}-5), 2.37(2 \mathrm{H}, \mathrm{q}, J \mathrm{~J} .6, \mathrm{H}-4), 2.07\left(1 \mathrm{H}, \mathrm{td}, J 11.4\right.$ and $\left.3.5, \mathrm{CHC}\left(\mathrm{CH}_{3}\right)_{2} \mathrm{Ph}\right)$, $1.80\left(2 \mathrm{H}, \mathrm{m}, \mathrm{CH}_{2} \mathrm{CHCH}_{3}\right), 1.67(1 \mathrm{H}, \mathrm{m}$, aliphatic $\mathrm{H}), 1.45(1 \mathrm{H}, \mathrm{m}$, aliphatic $\mathrm{H}), 1.25\left(3 \mathrm{H}, \mathrm{s}, \mathrm{CH}_{3} \mathrm{CCH}_{3} \mathrm{Ph}\right), 1.16(3 \mathrm{H}$, $\left.\mathrm{s}, \mathrm{CH}_{3} \mathrm{CCH}_{3} \mathrm{Ph}\right), 0.85\left(3 \mathrm{H}, \mathrm{s}, \mathrm{CH}_{3} \mathrm{CH}\right)$ and 0.8-1.35 (3H, m, aliphatic $\left.\mathrm{H}\right), \mathrm{NH}$ not visible; $\delta_{\mathrm{C}}\left(75 \mathrm{MHz}, \mathrm{CDCl}_{3}\right) 163.3$, 153.9, 152.2, 141.2, 137.2, 136.3, 128.5, 128.4, 128.4, 128.1, 128.1, 128.0, 126.1, 125.7, 125.3, 124.9, 75.4, 67.0, $50.3,41.4,39.4,34.5,34.2,31.3,30.2,29.2,26.4,23.5$ and $21.8 ; \mathrm{m} / \mathrm{z}$ (low resolution, ES) $562.0\left(22 \%, \mathrm{M}^{+}+{ }^{23} \mathrm{Na}\right)$, $557.0\left(35 \%, \mathrm{M}^{+}+\mathrm{NH}_{4}{ }^{+}\right)$and $540.0\left(100 \%, \mathrm{M}^{+}+\mathrm{H}\right) ; \mathrm{m} / \mathrm{z}$ (accurate mass, ES) Found: $\mathrm{M}^{+}+\mathrm{Na}, 562.2929 . \mathrm{C}_{35} \mathrm{H}_{41} \mathrm{NO}_{4} \mathrm{Na}$ requires $\mathrm{M}, 562.2933$.

\section{General method for asymmetric deconjugative alkylation of dehydroamino acid esters $\mathbf{5}$}

A flask charged with lithium chloride (6.0 equivalents) was flame dried under vacuum, allowed to cool to room temperature and transferred to a nitrogen atmosphere. THF and diisopropylamine ( 3.0 equivalents, $0.6 \mathrm{M}$ typical concentration) were added and the mixture cooled to $-78^{\circ} \mathrm{C}$ before a solution of $n$-butyllithium (3.0 equivalents, $\sim 1.6 \mathrm{M}$ in hexanes) was added. The reaction mixture was stirred for 20 minutes at $0{ }^{\circ} \mathrm{C}$, recooled to $-78^{\circ} \mathrm{C}$ and a solution of the (Z)-dehydroamino acid 5 (1.0 equivalent) in THF (0.2 M, typical concentration) was added dropwise. The reaction mixture was stirred for 20 minutes before alkyl halide (4.0 equivalents unless otherwise stated) was added. The reaction mixture was stirred for a further 7 hours (or until reaction was shown to be complete by TLC) at $-78{ }^{\circ} \mathrm{C}$ under nitrogen. The reaction mixture was quenched with a saturated aqueous ammonium chloride solution, diluted with water and the mixture extracted with ethyl acetate $(3 \times)$. The combined organic extracts were dried $\left(\mathrm{Na}_{2} \mathrm{SO}_{4}\right)$, filtered and evaporated under reduced pressure to give a crude product, which was purified by flash chromatography, typically eluting with petrol-diethyl ether, to give $\alpha, \alpha$-disubstituted amino acid 4 .

(2R,3E)-2-Benzyloxycarbonylamino-2-methyldodec-3-enoic acid (-)-8-phenylmenthyl ester 4a

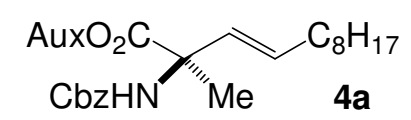

Colourless oil; $R_{\mathrm{F}} 0.44$ (20\% $\mathrm{Et}_{2} \mathrm{O}$ in petrol); $[\alpha]_{\mathrm{D}}^{20}-2.7$ (c. 0.70 in $\left.\mathrm{CHCl}_{3}\right) ; v_{\max } / \mathrm{cm}^{-1}\left(\mathrm{CHCl}_{3}\right.$ solution) 3418, 3360, 1724, 1496, 1455, 1264 and 1241; $\delta_{\mathrm{H}}\left(300 \mathrm{MHz}, \mathrm{CDCl}_{3}\right)$ 7.43-7.20 $(9 \mathrm{H}, \mathrm{m}, \mathrm{Ph}), 7.17-7.09(1 \mathrm{H}, \mathrm{m}, \mathrm{Ph}), 5.67(1 \mathrm{H}, \mathrm{dt}$, J 15.6 and 6.7, H-4), $5.52\left(1 \mathrm{H}, \mathrm{d}, J\right.$ 15.6, H-3), $5.32(1 \mathrm{H}, \mathrm{brs}, \mathrm{NH}), 5.11\left(1 \mathrm{H}, \mathrm{d}, J 12.3, \mathrm{CH}_{A} \mathrm{H}_{\mathrm{B}} \mathrm{Ph}(\mathrm{Cbz})\right), 5.02(1 \mathrm{H}, \mathrm{d}$, $J$ 12.3, $\left.\mathrm{CH}_{\mathrm{A}} \mathrm{H}_{B} \mathrm{Ph}(\mathrm{Cbz})\right), 4.82\left(1 \mathrm{H}, \mathrm{td}, \mathrm{J} 10.5\right.$ and 4.4, CHOCO), 2.08-1.87 (4H, m, H-5 and $\left.\mathrm{CH}_{2} \mathrm{CHOCO}\right), 1.50(3 \mathrm{H}$, $\mathrm{s}, \mathrm{Me}), 1.45-1.15(2 \mathrm{H}, \mathrm{m}$, aliphatic $\mathrm{H})$ and 1.10-0.70 $(10 \mathrm{H}, \mathrm{m}$, aliphatic $\mathrm{H}) ; \delta_{\mathrm{C}}\left(75 \mathrm{MHz}, \mathrm{CDCl}_{3}\right) 172.5,154.8,151.1$, 137.0, 132.8, 129.7, 128.9, 128.6, 128.5, 128.5, 126.0, 125.8, 77.7, 66.8, 60.4, 50.6, 41.6, 40.5, 34.9, 32.6, 32.3, 31.7, 29.9, 29.7, 29.7, 29.4, 29.3, 27.6, 25.5, 23.1, 23.1, 22.2 and 14.5; m/z (low resolution, ES) 593.2 (25\%, 
$\left.\mathrm{M}^{+}+\mathrm{NH}_{4}\right), 577.2\left(42 \%, \mathrm{M}^{+}+\mathrm{H}+{ }^{13} \mathrm{C}\right), 576.2\left(100 \%, \mathrm{M}^{+}+\mathrm{H}\right)$ and $362.0(29 \%) ; \mathrm{m} / \mathrm{z}$ (accurate mass, ES) Found: $\mathrm{M}^{+}+{ }^{23} \mathrm{Na}, 598.3890 . \mathrm{C}_{37} \mathrm{H}_{53} \mathrm{NO}_{4} \mathrm{Na}$ requires $\mathrm{M}, 598.3872$.

(2R,3E)-2-Benzyloxycarbonylamino-2-ethyldodec-3-enoic acid (-)-8-phenylmenthyl ester 4b

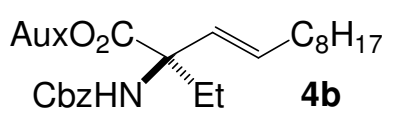

Colourless oil; $R_{\mathrm{F}} 0.57$ (20\% $\% \mathrm{Et}_{2} \mathrm{O}$ in petrol); $[\alpha]_{\mathrm{D}}^{26}-4.65$ (c. 0.71 in $\left.\mathrm{CHCl}_{3}\right) ; v_{\max } / \mathrm{cm}^{-1}\left(\mathrm{CHCl}_{3}\right.$ solution) 3420, 3369, 1718, 1495, 1455, 1244 and 1068; $\delta_{\mathrm{H}}\left(300 \mathrm{MHz} \mathrm{CDCl}_{3}\right)$ 7.42-7.21 $(9 \mathrm{H}, \mathrm{m}, \mathrm{Ph}), 7.19-7.10(1 \mathrm{H}, \mathrm{m}, \mathrm{Ph}), 5.70-5.57$ $(2 \mathrm{H}, \mathrm{m}, \mathrm{H}-4$ and $\mathrm{NH}), 5.45(1 \mathrm{H}, \mathrm{d}, J 15.4, \mathrm{H}-3), 5.13\left(1 \mathrm{H}, \mathrm{d}, J 12.3, \mathrm{CH}_{A} \mathrm{H}_{\mathrm{B}} \mathrm{Ph}(\mathrm{Cbz})\right), 5.03(1 \mathrm{H}, \mathrm{d}, J$ 12.3, $\left.\mathrm{CH}_{\mathrm{A}} \mathrm{H}_{B} \mathrm{Ph}(\mathrm{Cbz})\right), 4.82\left(1 \mathrm{H}, \mathrm{td}, \mathrm{J} 10.2\right.$ and 4.1, CHOCO), $2.25\left(1 \mathrm{H}, \mathrm{m}, \mathrm{CH}_{A} \mathrm{H}_{\mathrm{B}} \mathrm{CH}_{3}\right), 2.07-1.87(5 \mathrm{H}, \mathrm{m}, \mathrm{H}-5$, $\mathrm{CH}_{\mathrm{A}} \mathrm{H}_{B} \mathrm{CH}_{3}, \mathrm{CH}_{2} \mathrm{CHOCO}$ ) and 1.48-0.70 (33H, m, aliphatics); $\delta_{\mathrm{C}}\left(75 \mathrm{MHz}, \mathrm{CDCl}_{3}\right) 172.2,154.3,150.9,137.2,132.4$, 128.9, 128.5, 128.5, 128.5, 126.1, 126.1, 125.8, 77.8, 66.7, 64.5, 50.5, 41.7, 40.6, 34.8, 32.6, 32.3, 31.7, 30.1, 29.9, 29.7, 29.7, 29.4, 28.3, 27.7, 25.0, 23.1, 22.2, 14.5 and 8.5; $\mathrm{m} / \mathrm{z}$ (low resolution, ES) $1197.5\left(25 \%, 2 \mathrm{M}^{+}+\mathrm{NH}_{4}{ }^{+}+{ }^{13} \mathrm{C}\right)$, $1196.6\left(30 \%, 2 \mathrm{M}^{+}+\mathrm{NH}_{4}{ }^{+}\right), 1179.6\left(18 \%, 2 \mathrm{M}^{+}+\mathrm{H}\right), 607.3\left(25 \%, \mathrm{M}^{+}+\mathrm{NH}_{4}{ }^{+}\right), 591.2\left(42 \%, \mathrm{M}^{+}+\mathrm{H}_{+}{ }^{13} \mathrm{C}\right)$ and $590.1(100 \%$, $\mathrm{M}^{+}+\mathrm{H}$ ); $\mathrm{m} / \mathrm{z}$ (accurate mass, ES) Found: $\mathrm{M}^{+}+\mathrm{H}, 590.4200 . \mathrm{C}_{38} \mathrm{H}_{56} \mathrm{NO}_{4}$ requires $\mathrm{M}, 590.4209$.

(2R,3E)-2-Benzyl-2-benzyloxycarbonylaminododec-3-enoic acid (-)-8-phenylmenthyl ester 4c

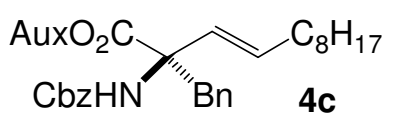

Colourless oil; $R_{\mathrm{F}} 0.43$ (15\% $\mathrm{Et}_{2} \mathrm{O}$ in petrol); $[\alpha]_{\mathrm{D}}^{22}+15.04$ (c. 0.645 in $\left.\mathrm{CHCl}_{3}\right) ; v_{\max } / \mathrm{cm}^{-1}\left(\mathrm{CHCl}_{3}\right.$ solution) 3415 , 1725, 1496, 1455, 1206, 1073 and 1022; $\delta_{\mathrm{H}}\left(300 \mathrm{MHz}, \mathrm{CDCl}_{3}\right)$ 7.46-7.27 (5H, m, Ph), 7.23-7.01 (10H, m, Ph), 5.60 $\left(1 \mathrm{H}, \mathrm{dt}, J 15.4\right.$ and 6.7, H-4), $5.47(1 \mathrm{H}, \mathrm{d}, J 15.4, \mathrm{H}-3), 5.22(1 \mathrm{H}, \mathrm{brs}, \mathrm{NH}), 5.11\left(2 \mathrm{H}, \mathrm{s}, \mathrm{CH}_{2} \mathrm{Ph}(\mathrm{Cbz})\right), 4.80(1 \mathrm{H}, \mathrm{td}, J$ 10.8 and 4.1, CHOCO), $3.36\left(1 \mathrm{H}, \mathrm{d}, J\right.$ 13.8, $\left.\mathrm{CH}_{A} \mathrm{H}_{\mathrm{B}} \mathrm{Ph}\right), 3.23\left(1 \mathrm{H}, \mathrm{d}, J 13.8, \mathrm{CH}_{\mathrm{A}} \mathrm{H}_{B} \mathrm{Ph}\right), 2.03(3 \mathrm{H}, \mathrm{m}, \mathrm{H}-5 \mathrm{and}$ $\left.\mathrm{CH}_{2} \mathrm{CHOCO}\right), 1.89\left(1 \mathrm{H}\right.$, brd, $\left.J 11.3, \mathrm{CH}_{2} \mathrm{CHOCO}\right)$ and 1.60-0.71 $\left(30 \mathrm{H}, \mathrm{m}\right.$, aliphatics); $\delta_{\mathrm{C}}\left(75 \mathrm{MHz}, \mathrm{CDCl}_{3}\right) 171.1$, 154.7, 151.3, 137.2, 136.7, 133.0, 131.1, 129.4, 128.9, 128.7, 128.5, 128.5, 128.4, 127.1, 125.9, 125.4, 78.3, 66.7, 64.9, 50.4, 41.6, 40.3, 40.1, 34.9, 32.6, 32.3, 31.8, 29.9, 29.7, 29.7, 29.4, 28.2, 27.7, 26.1, 23.1, 22.1 and 14.6; $\mathrm{m} / \mathrm{z}$ (low resolution, ES) $669.2\left(15 \%, \mathrm{M}^{+}+\mathrm{NH}_{4}{ }^{+}\right), 653.3\left(42 \%, \mathrm{M}^{+}+\mathrm{H}+{ }^{13} \mathrm{C}\right), 652.2\left(87 \%, \mathrm{M}^{+}+\mathrm{H}\right), 144.7(78 \%)$ and 114.8 $(100 \%) ; \mathrm{m} / \mathrm{z}$ (accurate mass, ES) Found: $\mathrm{M}^{+}+\mathrm{H}, 652.4359 . \mathrm{C}_{43} \mathrm{H}_{58} \mathrm{NO}_{4}$ requires $\mathrm{M}, 652.4366$.

(2R,3E)-2-Allyl-2-benzyloxycarbonylaminododec-3-enoic acid (-)-8-phenylmenthyl ester 4d

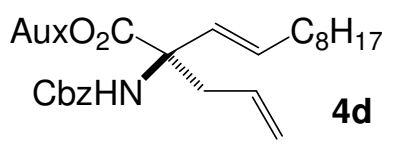

Colourless oil; $R_{\mathrm{F}} 0.39$ (15\% $\mathrm{Et}_{2} \mathrm{O}$ in petrol); $[\alpha]_{\mathrm{D}}{ }^{20}+3.74$ (c. 0.695 in $\left.\mathrm{CHCl}_{3}\right) ; v_{\max } / \mathrm{cm}^{-1}\left(\mathrm{CHCl}_{3}\right.$ solution) 3420 , 1719, 1495, 1467, 1221, 1086 and 1071; $\delta_{\mathrm{H}}\left(300 \mathrm{MHz}, \mathrm{CDCl}_{3}\right) 7.40-7.23(9 \mathrm{H}, \mathrm{m}, \mathrm{Ph}), 7.15(1 \mathrm{H}, \mathrm{m}, \mathrm{Ph}), 5.65(1 \mathrm{H}$, $\mathrm{dt}, J 15.4$ and 6.7, $\mathrm{H}-4), 5.62-5.50\left(2 \mathrm{H}, \mathrm{m}, \mathrm{CH}_{2} \mathrm{CH}=\mathrm{CH}_{2}\right.$ and $\left.\mathrm{NH}\right), 5.46(1 \mathrm{H}, \mathrm{d}, J 15.4, \mathrm{H}-3), 5.15-5.00(4 \mathrm{H}, \mathrm{m}$, $\mathrm{CH}_{2} \mathrm{Ph}(\mathrm{Cbz})$ and $\left.\mathrm{CH}_{2} \mathrm{CH}=\mathrm{CH}_{2}\right), 4.82(1 \mathrm{H}, \mathrm{td}, \mathrm{J} 10.8$ and 4.1, $\mathrm{CHOCO}), 2.93(1 \mathrm{H}$, dd, $\mathrm{J} 13.8$ and 6.7, $\left.\mathrm{CH}_{A} \mathrm{H}_{\mathrm{B}} \mathrm{CH}=\mathrm{CH}_{2}\right), 2.60\left(1 \mathrm{H}\right.$, dd, $\mathrm{J} 13.8$ and 7.2, $\left.\mathrm{CH}_{\mathrm{A}} \mathrm{H}_{B} \mathrm{CH}=\mathrm{CH}_{2}\right), 1.98\left(4 \mathrm{H}, \mathrm{m}, \mathrm{H}-5\right.$ and $\left.\mathrm{CH}_{2} \mathrm{CHOCO}\right), 1.59-1.15$ $(21 \mathrm{H}, \mathrm{m}$, aliphatic $\mathrm{H})$ and 1.13-0.68 $(9 \mathrm{H}, \mathrm{m}$, aliphatic $\mathrm{H}) ; \delta_{\mathrm{C}}\left(75 \mathrm{MHz}, \mathrm{CDCl}_{3}\right) 171.8,154.4,151.0,137.2,132.9$, 132.5, 128.9, 128.5, 128.4, 128.3, 126.1, 125.9, 125.8, 119.8, 78.0, 66.7, 63.3, 50.5, 41.9, 40.6, 39.6, 34.8, 32.6, $32.3,31.7,30.0,29.9,29.7,29.7,29.4,27.7,25.0,23.1,22.2$ and 14.5; m/z (low resolution, ES) 619.3 (18\%, 
$\left.\mathrm{M}^{+}+\mathrm{NH}_{4}{ }^{+}\right), 603.2\left(43 \%, \mathrm{M}^{+}+\mathrm{H}+{ }^{13} \mathrm{C}\right), 602.3\left(100 \%, \mathrm{M}^{+}+\mathrm{H}\right)$ and $388.1(53 \%) ; \mathrm{m} / \mathrm{z}$ (accurate mass, ES) Found: $\mathrm{M}^{+}+\mathrm{H}$, 602.4211. $\mathrm{C}_{39} \mathrm{H}_{56} \mathrm{NO}_{4}$ requires $\mathrm{M}, 602.4209$.

(2S,3E)-2-Benzyloxycarbonylamino-2-benzyloxymethyldodec-3-enoic acid (-)-8-phenylmenthyl ester 4e

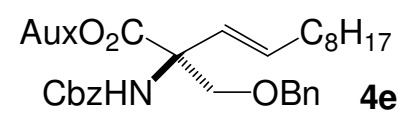

(NB - The product was further purified by preparative HPLC to give an analytical sample, for which the overall yield is $35 \%$ ) Colourless oil; $R_{\mathrm{F}} 0.29$ (15\% $\mathrm{Et}_{2} \mathrm{O}$ in petrol); $[\alpha]_{\mathrm{D}}^{21}-19.5$ (c. 0.2 in $\left.\mathrm{CHCl}_{3}\right) ; v_{\max } / \mathrm{cm}^{-1}\left(\mathrm{CHCl}_{3}\right.$ solution) 3421 , 1724, 1496, 1454, 1241, 1093 and 1056; $\delta_{\mathrm{H}}\left(300 \mathrm{MHz}, \mathrm{CDCl}_{3}\right) 7.43-7.08(15 \mathrm{H}, \mathrm{m}, \mathrm{Ph}), 5.90(1 \mathrm{H}, \mathrm{s}, \mathrm{NH}), 5.73(1 \mathrm{H}$, dt, J 15.9 and 6.7, H-4), $5.40\left(1 \mathrm{H}, \mathrm{d}, J\right.$ 15.9, H-3), $5.12\left(1 \mathrm{H}, \mathrm{d}, J\right.$ 12.8, $\left.\mathrm{CH}_{A} \mathrm{H}_{\mathrm{B}} \mathrm{Ph}(\mathrm{Cbz})\right), 5.05(1 \mathrm{H}, \mathrm{d}, J 12.3$, $\left.\mathrm{CH}_{\mathrm{A}} \mathrm{H}_{B} \mathrm{Ph}(\mathrm{Cbz})\right), 4.84\left(1 \mathrm{H}\right.$, td, J 10.8 and 4.1, CHOCO), $4.52\left(1 \mathrm{H}, \mathrm{d}, J 12.3, \mathrm{CH}_{A} \mathrm{H}_{\mathrm{B}} \mathrm{Ph}\right), 4.44(1 \mathrm{H}, \mathrm{d}, J 12.3$, $\left.\mathrm{CH}_{\mathrm{A}} H_{B} \mathrm{Ph}\right), 4.00\left(1 \mathrm{H}, \mathrm{d}, J\right.$ 9.2, $\left.\mathrm{CH}_{A} \mathrm{H}_{\mathrm{B}} \mathrm{OBn}\right), 3.80\left(1 \mathrm{H}, \mathrm{d}, J\right.$ 9.2, $\left.\mathrm{CH}_{\mathrm{A}} \mathrm{H}_{B} \mathrm{OBn}\right), 2.10-1.78(4 \mathrm{H}, \mathrm{m}$, aliphatic H), 1.55$1.15(21 \mathrm{H}, \mathrm{m}$, aliphatic $\mathrm{H})$ and 0.98-0.67 $(9 \mathrm{H}, \mathrm{m}$, aliphatic $\mathrm{H}) ; \delta_{\mathrm{C}}\left(75 \mathrm{MHz}, \mathrm{CDCl}_{3}\right) 169.4,153.3,149.4,136.8,135.8$, 132.6, 127.4, 127.2, 127.0, 127.0, 126.9, 126.7, 126.6, 124.7, 124.4, 123.7, 76.6, 72.4, 70.5, 65.3, 63.3, 49.2, 40.2, 39.2, 33.4, 31.2, 30.8, 30.2, 29.0, 28.4, 28.2, 27.8, 26.3, 26.2, 22.9, 21.6, 20.6 and 13.1; $\mathrm{m} / \mathrm{z}$ (low resolution, ES); $683.0\left(22 \%, \mathrm{M}^{+}+\mathrm{H}+{ }^{13} \mathrm{C}\right)$ and $682.1\left(39 \%, \mathrm{M}^{+}+\mathrm{H}\right) ; \mathrm{m} / \mathrm{z}$ (accurate mass, ES) Found: $\mathrm{M}^{+}+\mathrm{H}, 682.4469 . \mathrm{C}_{44} \mathrm{H}_{60} \mathrm{NO}_{5}$ requires $\mathrm{M}, 682.4471$.

(2R,1'E)-2-Benzyloxycarbonylamino-2-dec-1'-enylsuccinic acid 4-t-butyl, 1-[(-)-8-phenylmenthyl] diester 4f

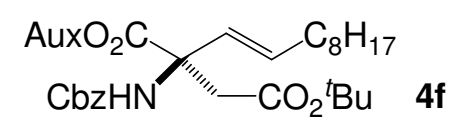

Colourless oil; $R_{\mathrm{F}} 0.55$ (20\% $\mathrm{Et}_{2} \mathrm{O}$ in petrol); $[\alpha]_{\mathrm{D}}^{26}-9.88$ (c. 4.23 in $\left.\mathrm{CHCl}_{3}\right) ; v_{\max } / \mathrm{cm}^{-1}\left(\mathrm{CHCl}_{3}\right.$ solution) 3422, 1738, 1496, 1455, 1368, 1155 and 1027; $\delta_{\mathrm{H}}\left(300 \mathrm{MHz} \mathrm{CDCl}_{3}\right) 7.40-7.15(10 \mathrm{H}, \mathrm{m}, \mathrm{Ph}), 6.26(1 \mathrm{H}, \mathrm{s}, \mathrm{NH}), 5.74(1 \mathrm{H}, \mathrm{dt}, J$ 15.6 and 6.7, $\left.\mathrm{H}-2^{\prime}\right), 5.37\left(1 \mathrm{H}, \mathrm{d}, J 15.6, \mathrm{H}-1^{\prime}\right), 5.10\left(2 \mathrm{H}, \mathrm{s}, \mathrm{CH}_{2} \mathrm{Ph}(\mathrm{Cbz})\right), 4.90(1 \mathrm{H}, \mathrm{td}, J 10.8$ and 4.1, CHOCO), $3.39\left(1 \mathrm{H}, \mathrm{d}, J \mathrm{~J} 16.9, \mathrm{H}-3_{\mathrm{A}}\right), 2.85\left(1 \mathrm{H}, \mathrm{d}, J 16.6, \mathrm{H}-3_{\mathrm{B}}\right), 2.20-1.90(4 \mathrm{H}, \mathrm{m}$, aliphatic $\mathrm{H})$ and 1.65-0.70 (39H, m, aliphatic $\mathrm{H}) ; \delta_{\mathrm{C}}\left(75 \mathrm{MHz}, \mathrm{CDCl}_{3}\right) 171.3,169.8,154.4,151.0,137.2,133.7,128.8,128.5,128.3,128.2,127.5,126.1,125.8$, 81.5, 78.3, 66.6, 61.2, 50.6, 41.5, 40.6, 40.2, 34.9, 32.5, 32.3, 31.7, 30.2, 29.8, 29.7, 29.2, 28.5, 28.1, 27.8, 24.8, 23.1, 22.1 and 14.5; $\mathrm{m} / \mathrm{z}$ (low resolution, ES) $698.4\left(82 \%, \mathrm{M}^{+}+{ }^{23} \mathrm{Na}\right.$ ) and $699.4\left(42 \%, \mathrm{M}^{+}+{ }^{23} \mathrm{Na}+{ }^{13} \mathrm{C}\right) ; \mathrm{m} / \mathrm{z}$ (accurate mass, ES) Found: $\mathrm{M}^{+}+{ }^{23} \mathrm{Na}, 698.4389 . \mathrm{C}_{42} \mathrm{H}_{61} \mathrm{NO}_{6}{ }^{23} \mathrm{Na}$ requires $\mathrm{M}, 698.4397$.

\section{(2R,3E)-2-Benzyloxycarbonylamino-2-methyl-4-phenylbut-3-enoic acid (-)-8-phenylmenthyl ester $4 \mathrm{~g}$}

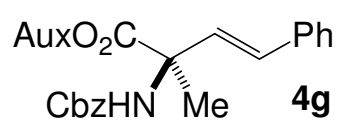

Colourless oil; $R_{\mathrm{F}} 0.42$ (30\% $\mathrm{Et} \mathrm{t}_{2} \mathrm{O}$ in petrol); $[\alpha]_{\mathrm{D}}^{22}+6.88$ (c. 0.48 in $\left.\mathrm{CHCl}_{3}\right) ; v_{\max } / \mathrm{cm}^{-1}\left(\mathrm{CHCl}_{3}\right.$ solution) 3414, 3367, 1721, 1496, 1454, 1260 and 1058; $\delta_{\mathrm{H}}\left(300 \mathrm{MHz}, \mathrm{CDCl}_{3}\right)$ 7.45-7.07 $(15 \mathrm{H}, \mathrm{m}, \mathrm{Ph}), 6.54(1 \mathrm{H}, \mathrm{d}, J$ 16.4, H-4), $6.34(1 \mathrm{H}$, d, J 16.4, H-3), $5.43\left(1 \mathrm{H}\right.$, brs, NH), $5.14\left(1 \mathrm{H}, \mathrm{d}, J\right.$ 12.3, $\left.\mathrm{CH}_{A} \mathrm{H}_{\mathrm{B}} \mathrm{Ph}(\mathrm{Cbz})\right), 5.04\left(1 \mathrm{H}, \mathrm{d}, J\right.$ 12.3, $\left.\mathrm{CH}_{\mathrm{A}} \mathrm{H}_{B} \mathrm{Ph}(\mathrm{Cbz})\right), 4.84$ $(1 \mathrm{H}, \mathrm{td}, \mathrm{J} 10.8$ and $4.1, \mathrm{CHOCO}), 1.88\left(2 \mathrm{H}, \mathrm{m}, \mathrm{CH}_{2} \mathrm{CHOCO}\right)$ and 1.70-0.70 $(18 \mathrm{H}, \mathrm{m}$, aliphatic $\mathrm{H})$, signal visible for minor diastereoisomer at $6.10(1 \mathrm{H}, \mathrm{d}, J 15.9, \mathrm{H}-3) ; \delta_{\mathrm{C}}\left(75 \mathrm{MHz}, \mathrm{CDCl}_{3}\right) 172.3,154.8,151.1,137.0,136.6,130.9$, 129.5, 128.9, 128.9, 128.7, 128.5, 128.2, 127.1, 126.0, 125.8, 125.7, 77.8, 67.0, 60.7, 50.5, 41.6, 40.5, 34.9, 31.7, 28.8, 27.5, 25.9, 23.7 and 22.2; $\mathrm{m} / \mathrm{z}$ (low resolution, ES) $562.2\left(25 \%, \mathrm{M}^{+}+\mathrm{Na}\right), 557.2\left(55 \%, \mathrm{M}^{+}+\mathrm{NH}_{4}{ }^{+}\right), 540.2(70 \%$, 
$\mathrm{M}^{+}+\mathrm{H}$ ) and $389.1(100 \%) ; \mathrm{m} / \mathrm{z}$ (accurate mass, ES) Found: $\mathrm{M}^{+}+{ }^{23} \mathrm{Na}, 562.2927 . \mathrm{C}_{35} \mathrm{H}_{41} \mathrm{NO}_{4}{ }^{23} \mathrm{Na}$ requires $\mathrm{M}$, 562.2933 .

(2S,3E)-2-Benzyloxycarbonylamino-2-benzyloxymethyl-4-phenylbut-3-enoic (-)-8-phenylmenthyl ester 4h

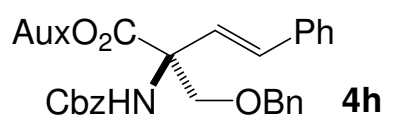

Colourless oil; $R_{\mathrm{F}} 0.46$ (30\% $\mathrm{Et}_{2} \mathrm{O}$ in petrol); $[\alpha]_{\mathrm{D}}^{26}-26.02$ (c. 0.42 in $\left.\mathrm{CHCl}_{3}\right) ; v_{\max } / \mathrm{cm}^{-1}\left(\mathrm{CHCl}_{3}\right.$ solution) 3418, $1721,1599,1495,1453,1241,1053$ and 697; $\delta_{\mathrm{H}}\left(300 \mathrm{MHz}, \mathrm{CDCl}_{3}\right)$ 7.47-7.07 $(20 \mathrm{H}, \mathrm{m}, \mathrm{Ph}), 6.62(1 \mathrm{H}, \mathrm{d}, J 15.9, \mathrm{H}-$ 4), $6.22(1 \mathrm{H}, \mathrm{d}, J 16.4, \mathrm{H}-3), 6.02(1 \mathrm{H}, \mathrm{brs}, \mathrm{NH}), 5.16\left(1 \mathrm{H}, \mathrm{d}, J 12.8, \mathrm{CH}_{A} \mathrm{H}_{\mathrm{B}} \mathrm{Ph}(\mathrm{Cbz})\right), 5.06(1 \mathrm{H}, \mathrm{d}, J 12.3$, $\left.\mathrm{CH}_{\mathrm{A}} \mathrm{H}_{B} \mathrm{Ph}(\mathrm{Cbz})\right), 4.86\left(1 \mathrm{H}, \mathrm{td}, J 10.8\right.$ and 4.6, CHOCO), $4.55\left(1 \mathrm{H}, \mathrm{d}, J 11.8, \mathrm{CH}_{A} \mathrm{H}_{\mathrm{B}} \mathrm{Ph}\right), 4.48(1 \mathrm{H}, \mathrm{d}, \mathrm{J} 11.8$, $\left.\mathrm{CH}_{\mathrm{A}} \mathrm{H}_{B} \mathrm{Ph}\right), 4.06\left(1 \mathrm{H}\right.$, brd, J 9.2, $\left.\mathrm{CH}_{A} \mathrm{H}_{\mathrm{B}} \mathrm{OBn}\right), 3.89\left(1 \mathrm{H}\right.$, brd, J 9.2, $\left.\mathrm{CH}_{\mathrm{A}} \mathrm{H}_{B} \mathrm{OBn}\right), 2.04(1 \mathrm{H}$, brd, J 10.2, aliphatic $\mathrm{H})$, $1.84(1 \mathrm{H}, \mathrm{dt}, J 11.8$ and 3.1, aliphatic $\mathrm{H})$ and 1.52-0.60 $(15 \mathrm{H}, \mathrm{m}$, aliphatic $\mathrm{H}) ; \delta_{\mathrm{C}}\left(75 \mathrm{MHz}, \mathrm{CDCl}_{3}\right) 170.6,154.9$, $150.7,138.0,137.2,136.5,132.2,128.9,128.9,128.8,128.6,128.5,128.4,128.4,128.2,128.2,127.1,126.1$, 125.8, 125.1, 78.4, 74.0, 72.2, 66.9, 65.1, 50.8, 41.7, 40.7, 34.8, 31.7, 30.7, 27.8, 24.1, 22.1; m/z (low resolution, ES) $669.1\left(33 \%, \mathrm{M}^{+}+{ }^{23} \mathrm{Na}+{ }^{13} \mathrm{C}\right), 668.1\left(71 \%, \mathrm{M}^{+}+{ }^{23} \mathrm{Na}\right)$ and $646.1\left(69 \%, \mathrm{M}^{+}+\mathrm{H}\right) ; \mathrm{m} / \mathrm{z}$ (accurate mass, ES) Found: $\mathrm{M}^{+}+\mathrm{H}, 646.3553 . \mathrm{C}_{42} \mathrm{H}_{48} \mathrm{NO}_{5}$ requires $\mathrm{M}, 646.3532$.

(2R,3E)-Benzyloxycarbonylamino-2-methyl-5-phenylpent-3-enoic acid (-)-8-phenylmenthyl ester 4i

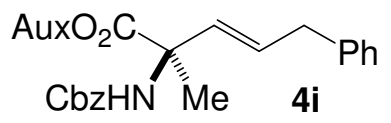

Colourless oil; $R_{\mathrm{F}} 0.46$ (30\% $\mathrm{Et}_{2} \mathrm{O}$ in petrol); $[\alpha]_{\mathrm{D}}^{22}+6.74$ (c. 0.445 in $\left.\mathrm{CHCl}_{3}\right) ; v_{\max } / \mathrm{cm}^{-1}\left(\mathrm{CHCl}_{3}\right.$ solution) 3414, 3368, 1722, 1495, 1453, 1258, 1241 and 1050; $\delta_{\mathrm{H}}\left(300 \mathrm{MHz}, \mathrm{CDCl}_{3}\right)$ 7.42-7.08 (15H, m, Ph), $5.81(1 \mathrm{H}, \mathrm{dt}, J 15.4$ and 6.7, H-4), $5.62(1 \mathrm{H}, \mathrm{d}, J 15.8, \mathrm{H}-3), 5.28(1 \mathrm{H}, \mathrm{brs}, \mathrm{NH}), 5.11\left(1 \mathrm{H}, \mathrm{d}, J 11.8, \mathrm{CH}_{A} \mathrm{H}_{B} \mathrm{Ph}(\mathrm{Cbz})\right), 5.01(1 \mathrm{H}, \mathrm{d}, J 11.8$, $\left.\mathrm{CH}_{\mathrm{A}} \mathrm{H}_{B} \mathrm{Ph}(\mathrm{Cbz})\right), 4.83\left(1 \mathrm{H}, \mathrm{td}, J 10.8\right.$ and 4.6, CHOCO), $3.36(2 \mathrm{H}, \mathrm{d}, J 6.2, \mathrm{H}-5), 1.92\left(2 \mathrm{H}, \mathrm{m}, \mathrm{CH}_{2} \mathrm{CHOCO}\right)$ and 1.60-0.69 (18H, m, aliphatic $\mathrm{H}) ; \delta_{\mathrm{C}}\left(75 \mathrm{MHz}, \mathrm{CDCl}_{3}\right)$ 172.3, 154.8, 151.2, 140.0, 136.9, 131.4, 130.9, 129.0, 128.9, 128.8, 128.6, 128.5, 128.2, 126.6, 126.0, 125.8, 77.5, 66.9, 60.4, 50.4, 41.6, 40.5, 38.8, 34.9, 31.7, 28.7, 27.5, 25.9, 23.4 and 22.2; $\mathrm{m} / \mathrm{z}$ (low resolution, ES) $576.1\left(10 \%, \mathrm{M}^{+}+\mathrm{Na}\right), 571.2\left(57 \%, \mathrm{M}^{+}+\mathrm{NH}_{4}{ }^{+}\right), 554.1\left(100 \%, \mathrm{M}^{+}+\mathrm{H}\right)$ and 340.0 (47\%); $\mathrm{m} / \mathrm{z}$ (accurate mass, ES) Found: $\mathrm{M}^{+}+{ }^{23} \mathrm{Na}, 576.3105 . \mathrm{C}_{36} \mathrm{H}_{43} \mathrm{NO}_{4}{ }^{23} \mathrm{Na}$ requires $\mathrm{M}, 576.3090$.

\section{(2S,3E)-2-Benzyloxycarbonylamino-2-benzyloxymethyl-5-phenylpent-3-enoic (-)-8-phenylmenthyl ester 4j}

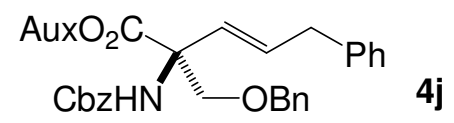

Colourless oil isolated in $>95 \%$ purity determined by ${ }^{1} \mathrm{H}-\mathrm{NMR}$ analysis; $R_{\mathrm{F}} 0.5$ (30\% $\mathrm{Et}_{2} \mathrm{O}$ in petrol); $[\alpha]_{\mathrm{D}}{ }^{26}-4.83$ (c. 0.12 in $\left.\mathrm{CHCl}_{3}\right) ; v_{\max } / \mathrm{cm}^{-1}\left(\mathrm{CHCl}_{3}\right.$ solution) 3414, 1722, 1628, 1601, 1241, 1084 and 1062; $\delta_{\mathrm{H}}\left(300 \mathrm{MHz}, \mathrm{CDCl}_{3}\right)$ 7.45-7.05 (20H, m, Ph), $5.90(1 \mathrm{H}, \mathrm{dt}, J 15.4$ and 6.7, H-4), $5.87(1 \mathrm{H}, \mathrm{brs}, \mathrm{NH}), 5.49(1 \mathrm{H}, \mathrm{d}, J 15.4, \mathrm{H}-3), 5.12(1 \mathrm{H}, \mathrm{d}$, $J$ 12.3, $\left.\mathrm{CH}_{A} \mathrm{H}_{\mathrm{B}} \mathrm{Ph}(\mathrm{Cbz})\right), 5.04\left(1 \mathrm{H}, \mathrm{d}, J 12.3, \mathrm{CH}_{\mathrm{A}} H_{B} \mathrm{Ph}(\mathrm{Cbz})\right), 4.84(1 \mathrm{H}, \mathrm{td}, J 10.8$ and 4.1, CHOCO), $4.50(1 \mathrm{H}, \mathrm{d}, J$ 12.3, $\left.\mathrm{OCH}_{A} \mathrm{H}_{B} \mathrm{Ph}\right), 4.43\left(1 \mathrm{H}, \mathrm{d}, J 11.8, \mathrm{OCH}_{\mathrm{A}} \mathrm{H}_{B} \mathrm{Ph}\right), 3.97\left(1 \mathrm{H}, \mathrm{d}, J\right.$ 8.7, $\left.\mathrm{CH}_{A} \mathrm{H}_{B} \mathrm{OBn}\right), 3.78\left(1 \mathrm{H}, \mathrm{d}, J\right.$ 8.7, $\left.\mathrm{CH}_{\mathrm{A}} H_{B} \mathrm{OBn}\right)$, $3.36(2 \mathrm{H}, \mathrm{d}, J 6.1, \mathrm{H}-5), 1.99\left(1 \mathrm{H}, \mathrm{brd}, J 11.8, \mathrm{CH}_{A} \mathrm{H}_{\mathrm{B}} \mathrm{CHOCO}\right), 1.85\left(1 \mathrm{H}, \mathrm{dt}, J 3.1\right.$ and $\left.11.8, \mathrm{CH}_{\mathrm{A}} \mathrm{H}_{B} \mathrm{CHOCO}\right)$ and $1.60-0.60(15 \mathrm{H}, \mathrm{m}$, aliphatic $\mathrm{H}) ; \delta_{\mathrm{C}}\left(75 \mathrm{MHz}, \mathrm{CDCl}_{3}\right) 170.2,154.4,150.5,139.4,137.7,136.7,131.8,128.6,128.4$, 
128.4, 128.3, 128.0, 128.0, 127.7, 127.6, 126.6, 126.1, 125.7, 125.5, 125.4, 77.7, 73.4, 71.5, 66.3, 64.4, 50.1, 41.1, 40.2, 38.4, 34.4, 31.2, 29.7, 27.2, 24.2 and 21.7; $\mathrm{m} / \mathrm{z}$ (low resolution, ES) $660.0\left(25 \%, \mathrm{M}^{+}+\mathrm{H}\right.$ ); $\mathrm{m} / \mathrm{z}$ (accurate mass, ES) Found: $\mathrm{M}^{+}+\mathrm{H}, 660.3707 . \mathrm{C}_{43} \mathrm{H}_{50} \mathrm{NO}_{5}$ requires $\mathrm{M}, 660.3689$.

\section{Reduction of amino acid esters 4 to oxazolidinones 12}

Method 1: Lithium aluminium hydride (1M solution in THF, 4 equivalents) was added to a stirred solution of $\alpha, \alpha-$ disubstituted amino acid ( 1 equivalent) in THF at $0{ }^{\circ} \mathrm{C}$ under nitrogen. The reaction mixture was stirred for 1 hour at $0{ }^{\circ} \mathrm{C}$. The mixture was quenched cautiously with methanol and warmed to room temperature. Sodium borohydride (8 equivalents) was added and the reaction mixture stirred for 1 hour at room temperature. The reaction mixture was quenched with $1 \mathrm{M}$ aqueous sodium hydroxide solution and stirring continued for 24 hours. The mixture was diluted with water and extracted with ethyl acetate $(3 \times)$. The combined organic extracts were dried $\left(\mathrm{NaSO}_{4}\right)$, filtered and evaporated under reduced pressure to give a crude product, which was purified by flash chromatography, eluting with petrol-diethyl ether, to give the oxazolidinone.

Method 2: Powdered lithium borohydride (16 equivalents) was cautiously added to a stirred solution of $\alpha, \alpha-$ disubstituted amino acid (1 equivalent) in diethyl ether at room temperature under nitrogen. The reaction mixture was heated at reflux for 1 hour. The reaction mixture was cooled to room temperature and quenched with a $1 \mathrm{M}$ aqueous sodium hydroxide solution. The mixture was heated at reflux for 30 minutes. The reaction mixture was cooled to room temperature and excess diethyl ether was removed under reduced pressure. THF was added and the reaction mixture stirred for 24 hours at room temperature. The remaining THF was removed under reduced pressure and the residue diluted with water. The aqueous mixture was extracted with ethyl acetate $(3 \times)$. The combined organic extracts were dried $\left(\mathrm{NaSO}_{4}\right)$, filtered and evaporated under reduced pressure to give a crude product, which was purified by flash chromatography, eluting with petrol-diethyl ether, to give the oxazolidinone.

\section{(1'E,4R)-4-Methyl-4-dec-1'-enyloxazolidin-2-one 12a}

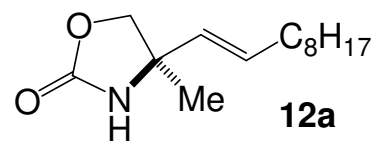

Colourless oil; $R_{\mathrm{F}} 0.45$ (60\% $\mathrm{Et}_{2} \mathrm{O}$ in petrol); $[\alpha]_{\mathrm{D}}{ }^{24}-14.38$ (c. 1.05 in $\mathrm{CHCl}_{3}$ ); (Found: C, 69.95; $\mathrm{H}, 10.7 ; \mathrm{N}, 5.9$; $\mathrm{C}_{14} \mathrm{H}_{25} \mathrm{NO}_{2}$ requires: $\left.\mathrm{C}, 70.25 ; \mathrm{H}, 10.53 ; \mathrm{N}, 5.85 \%\right) ; v_{\max } / \mathrm{cm}^{-1}\left(\mathrm{CHCl}_{3}\right.$ solution)3270, 1756, 1466, 1389, 1284 and $1041 ; \delta_{\mathrm{H}}\left(300 \mathrm{MHz}, \mathrm{CDCl}_{3}\right) 5.73\left(1 \mathrm{H}, \mathrm{dt}, J 15.6\right.$ and 6.7, H-2'), $5.61(1 \mathrm{H}, \mathrm{s}, \mathrm{NH}), 5.53\left(1 \mathrm{H}, \mathrm{d}, J 15.6, \mathrm{H}-1^{\prime}\right), 4.17(1 \mathrm{H}$, d, J 8.2, H-5 $\left.5_{\mathrm{A}}\right), 4.11\left(1 \mathrm{H}, \mathrm{d}, J\right.$ 8.2, $\left.\mathrm{H}-5_{\mathrm{B}}\right), 2.07\left(2 \mathrm{H}, \mathrm{q}, \mathrm{H}-3^{\prime}\right), 1.47(3 \mathrm{H}, \mathrm{s}, \mathrm{Me}), 1.45-1.23\left(12 \mathrm{H}, \mathrm{m}, \mathrm{CH}_{2} \times 6\right)$ and 0.92 $\left(3 \mathrm{H}, \mathrm{t}, \mathrm{J} 6.7, \mathrm{H}-10\right.$ '); $\delta_{\mathrm{C}}\left(75 \mathrm{MHz}, \mathrm{CDCl}_{3}\right)$ 159.4, 132.2, 132.0, 58.6, 32.5, 32.3, 30.7, 29.8, 29.7, 29.6, 29.4, 25.9, 23.1 and 14.5; $\mathrm{m} / \mathrm{z}$ (low resolution, ES) $281.1\left(100 \%, \mathrm{M}^{+}+\mathrm{CH}_{4}{ }^{+} \mathrm{CN}\right)$ and $257.1\left(93 \%, \mathrm{M}^{+}+\mathrm{NH}_{4}{ }^{+}\right)$. An enantiomeric excess of $95 \%$ was determined by chiral HPLC using a Daicel Chemical Industries Ltd. Chiralpak AS $(250 \times 4.6$ $\mathrm{mm}$ ) HPLC column, eluting with $10 \%$ IPA in hexane at a rate of $1 \mathrm{ml} / \mathrm{min}$ with retention times of 26 and 36 minutes. 
Representative HPLC chromatograms of racemic and enantiopure 12a:

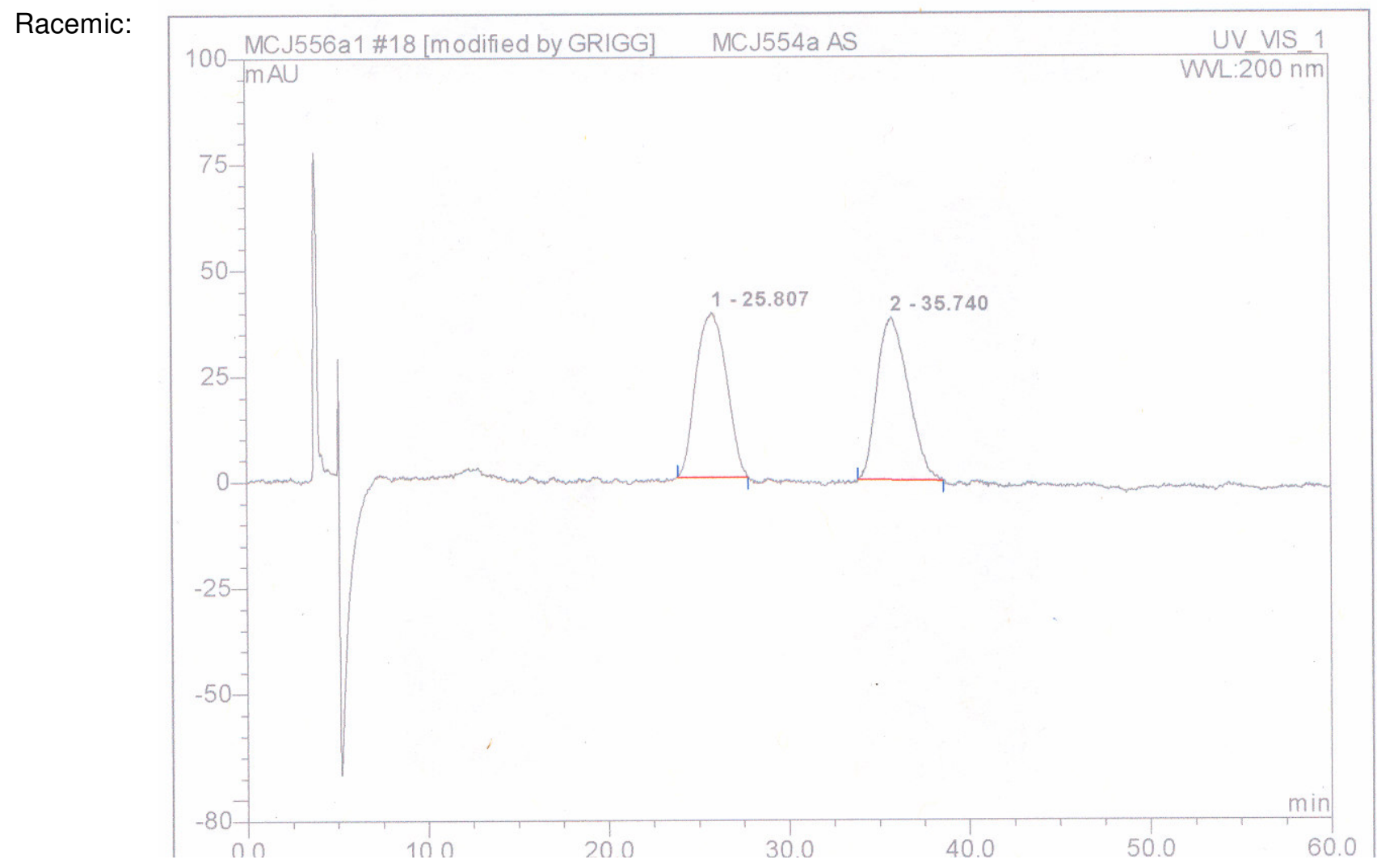

Enantioenriched:

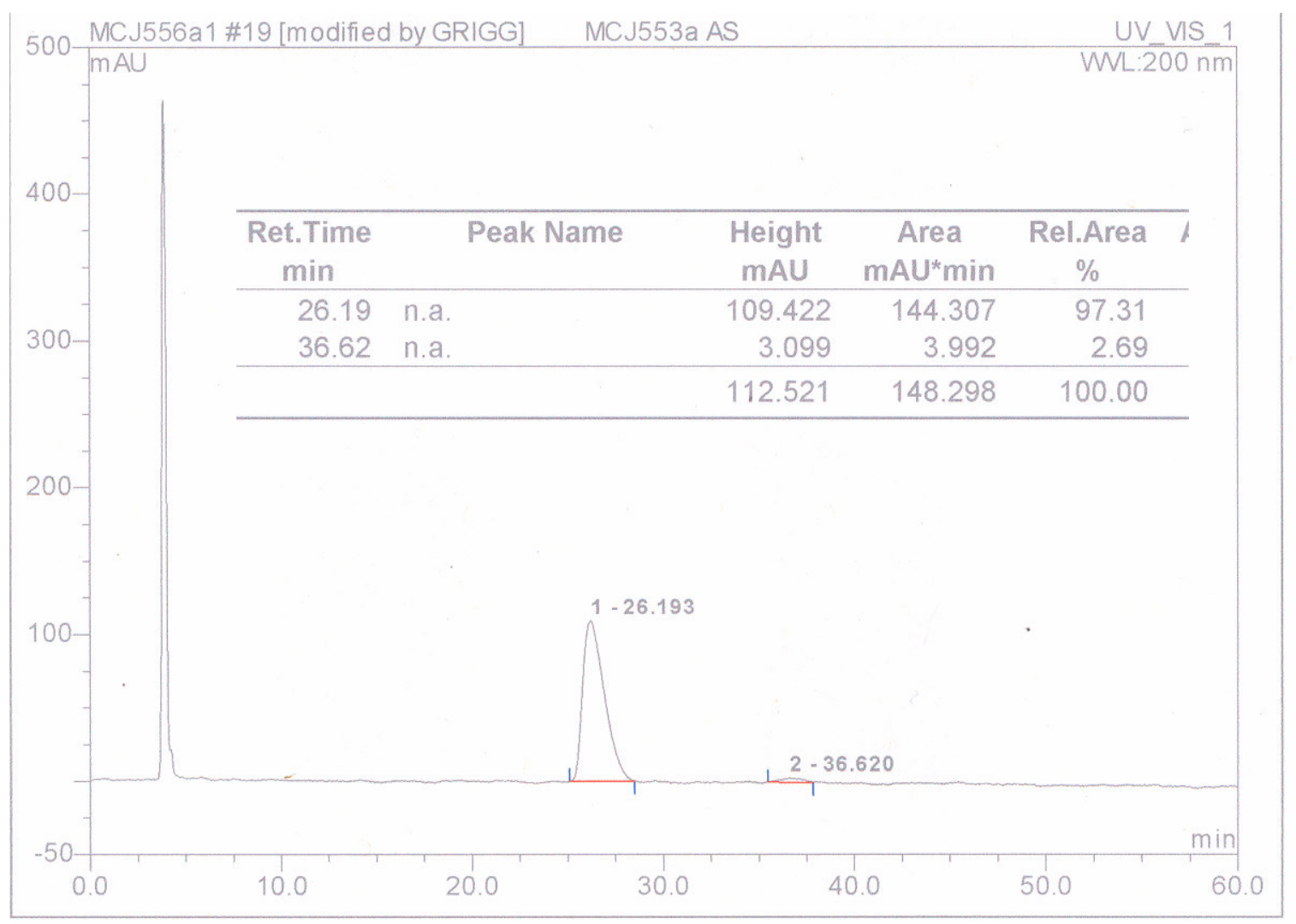




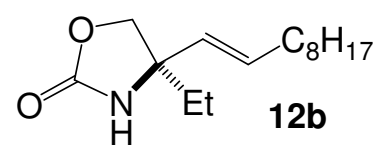

Colourless oil; $R_{\mathrm{F}} 0.47$ (60\% $\mathrm{Et}_{2} \mathrm{O}$ in petrol); $[\alpha]_{\mathrm{D}}{ }^{24}-26.84$ (c. 0.97 in $\mathrm{CHCl}_{3}$ ); (Found: C, 70.85; $\mathrm{H}, 10.75 ; \mathrm{N}, 5.65$; $\mathrm{C}_{15} \mathrm{H}_{27} \mathrm{NO}_{2}$ requires: $\left.\mathrm{C}, 71.10 ; \mathrm{H}, 10.74 ; \mathrm{N}, 5.53 \%\right) ; v_{\max } / \mathrm{cm}^{-1}$ ( $\mathrm{CHCl}_{3}$ solution) $3245,1755,1462,1393,1380$ and 1270; $\delta_{\mathrm{H}}\left(300 \mathrm{MHz}, \mathrm{CDCl}_{3}\right) 6.01(1 \mathrm{H}, \mathrm{s}, \mathrm{NH}), 5.69\left(1 \mathrm{H}, \mathrm{dt}, J 15.6\right.$ and 6.9, $\left.\mathrm{H}-2^{\prime}\right), 5.41\left(1 \mathrm{H}, \mathrm{dd}, J 15.6\right.$ and 1.3, $\left.\mathrm{H}-1^{\prime}\right)$, $4.16\left(1 \mathrm{H}, \mathrm{d}, J 8.7, \mathrm{H}-5_{\mathrm{A}}\right), 4.12\left(1 \mathrm{H}, \mathrm{d}, J 8.7, \mathrm{H}-5_{\mathrm{B}}\right), 2.05\left(2 \mathrm{H}, \mathrm{q}, J 6.9, \mathrm{H}-3^{\prime}\right), 1.70(1 \mathrm{H}, \mathrm{dq}, J 14.6$ and 7.4, $\left.\mathrm{CH}_{A} \mathrm{H}_{\mathrm{B}} \mathrm{CH}_{3}\right), 1.66\left(1 \mathrm{H}\right.$, dq, J 14.6 and 7.4, $\left.\mathrm{CH}_{\mathrm{A}} \mathrm{H}_{B} \mathrm{CH}_{3}\right), 1.43-1.20\left(12 \mathrm{H}, \mathrm{m}, \mathrm{CH}_{2} \times 6\right)$ and $0.90\left(6 \mathrm{H}, \mathrm{m}, \mathrm{CH}_{3} \times 2\right) ; \delta_{\mathrm{C}}$ $\left(75 \mathrm{MHz}, \mathrm{CDCl}_{3}\right)$ 159.8, 132.1, 131.3, 75.8, 62.0, 32.6, 32.5, 32.3, 29.8, 29.7, 29.5, 29.5, 23.1, 14.5 and 8.4; $\mathrm{m} / \mathrm{z}$ (low resolution, ES) $295.2\left(35 \%, \mathrm{M}^{+}+\mathrm{CH}_{4}{ }^{+} \mathrm{CN}\right)$ and $271.2\left(100 \%, \mathrm{M}^{+}+\mathrm{NH}_{4}{ }^{+}\right)$. An enantiomeric excess of $94 \%$ was determined by chiral HPLC using a Daicel Chemical Industries Ltd. Chiralpak AS $(250 \times 4.6 \mathrm{~mm})$ HPLC column, eluting with $10 \%$ IPA in hexane at a rate of $1 \mathrm{ml} / \mathrm{min}$ with retention times of 19 and 29 minutes.

\section{(4R,1'E)-4-Benzyl-4-dec-1'-enyloxazolidin-2-one 12c}

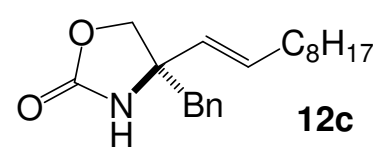

Colourless oil; $R_{\mathrm{F}} 0.24$ (50\% $\% \mathrm{Et}_{2} \mathrm{O}$ in petrol); $[\alpha]_{\mathrm{D}}^{24}-43.8$ (c. 0.32 in $\left.\mathrm{CHCl}_{3}\right) ; v_{\max } / \mathrm{cm}^{-1}\left(\mathrm{CHCl}_{3}\right.$ solution) 3255,1753 , 1392 and 1035; $\delta_{\mathrm{H}}\left(300 \mathrm{MHz}, \mathrm{CDCl}_{3}\right)$ 7.35-7.22 (3H, m, Ph), 7.18-7.11 (2H, m, Ph), $5.56(1 \mathrm{H}, \mathrm{dt}, J 15.9$ and 5.6, H2'), $5.48\left(1 \mathrm{H}, \mathrm{d}, J 15.9, \mathrm{H}-1^{\prime}\right), 5.23(1 \mathrm{H}, \mathrm{brs}, \mathrm{NH}), 4.31\left(1 \mathrm{H}, \mathrm{d}, J 8.2, \mathrm{H}-5_{\mathrm{A}}\right), 4.16\left(1 \mathrm{H}, \mathrm{d}, J 8.2, \mathrm{H}-5_{\mathrm{B}}\right), 2.97(1 \mathrm{H}, \mathrm{d}, J$ 13.3, $\left.\mathrm{CH}_{A} \mathrm{H}_{\mathrm{B}} \mathrm{Ph}\right), 2.91\left(1 \mathrm{H}, \mathrm{d}, J\right.$ 13.3, $\left.\left.\mathrm{CH}_{\mathrm{A}} \mathrm{H}_{B} \mathrm{Ph}\right), 2.03(2 \mathrm{H}, \mathrm{q}, J 6.7, \mathrm{H}-3)^{\prime}\right), 1.39-1.16\left(12 \mathrm{H}, \mathrm{m}, \mathrm{CH}_{2} \times 6\right)$ and $0.88(3 \mathrm{H}$, t, J 6.7, H-10'); $\delta_{\mathrm{C}}\left(75 \mathrm{MHz}, \mathrm{CDCl}_{3}\right)$ 158.5, 134.8, 132.1, 130.4, 130.3, 128.5, 127.3, 75.4, 61.6, 45.5, 32.1, 31.9, 29.4, 29.2, 29.1, 29.0, 22.7 and 14.1; m/z (low resolution, ES) $654.6\left(75 \%, 2 \mathrm{M}^{+}+{ }^{23} \mathrm{Na}+{ }^{13} \mathrm{C}\right), 653.6(100 \%$, $\left.2 \mathrm{M}^{+}+{ }^{23} \mathrm{Na}\right), 338.3\left(89 \%, \mathrm{M}^{+}+{ }^{23} \mathrm{Na}\right)$ and $316.3\left(65 \%, \mathrm{M}^{+}+\mathrm{H}\right) ; \mathrm{m} / \mathrm{z}$ (accurate mass, ES) Found: $\mathrm{M}^{+}+\mathrm{H}, 316.2282$. $\mathrm{C}_{20} \mathrm{H}_{30} \mathrm{NO}_{2}$ requires $\mathrm{M}, 316.2277$.An enantiomeric excess of $96 \%$ was determined by chiral HPLC using a Daicel Chemical Industries Ltd. Chiralpak AD $(250 \times 4.6 \mathrm{~mm})$ HPLC column, eluting with $3 \%$ IPA in hexane at a rate of 1 $\mathrm{ml} / \mathrm{min}$ with retention times of 15 and 25 minutes.

(1'E,4R)-4-Allyl-4-dec-1'-enyloxazolidin-2-one 12d

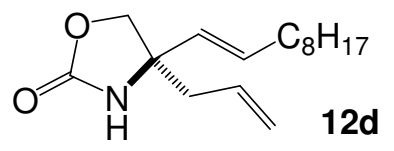

Colourless oil; $R_{\mathrm{F}} 0.23$ (50\% $\mathrm{Et}_{2} \mathrm{O}$ in petrol); $[\alpha]_{\mathrm{D}}^{24}-2.47$ (c. 0.85 in $\left.\mathrm{CHCl}_{3}\right) ; v_{\max } / \mathrm{cm}^{-1}\left(\mathrm{CHCl}_{3}\right.$ solution) 3350, 1754, 1478, 1423, 1380, 1346, 1130 and 1000; $\delta_{\mathrm{H}}\left(300 \mathrm{MHz}, \mathrm{CDCl}_{3}\right) 5.80-5.65\left(3 \mathrm{H}, \mathrm{m}, \mathrm{H}-2\right.$ ', $\mathrm{CH}_{2} \mathrm{CH}=\mathrm{CH}_{2}$ and $\left.\mathrm{NH}\right), 5.45$ $\left(1 \mathrm{H}, \mathrm{d}, J 15.6\right.$ and 1.3, $\left.\mathrm{H}-1^{\prime}\right), 5.25-5.15\left(2 \mathrm{H}, \mathrm{m}, \mathrm{CH}_{2} \mathrm{CH}=\mathrm{CH}_{2}\right), 4.20\left(1 \mathrm{H}, \mathrm{d}, J 8.5, \mathrm{H}-5_{\mathrm{A}}\right), 4.11\left(1 \mathrm{H}, \mathrm{d}, J 8.2, \mathrm{H}-5_{\mathrm{B}}\right)$, $2.42\left(2 \mathrm{H}, \mathrm{m}, \mathrm{CH}_{2} \mathrm{CH}=\mathrm{CH}_{2}\right), 2.05\left(2 \mathrm{H}, \mathrm{q}, \mathrm{J} 6.7, \mathrm{H}-3^{\prime}\right), 1.43-1.20\left(12 \mathrm{H}, \mathrm{m}, \mathrm{CH}_{2} \times 6\right)$ and $0.88\left(3 \mathrm{H}, \mathrm{t}, \mathrm{J} 6.7, \mathrm{H}-10^{\prime}\right) ; \delta_{\mathrm{C}}$ $\left(75 \mathrm{MHz}, \mathrm{CDCl}_{3}\right)$ 159.4, 132.5, 131.7, 131.0, 120.9, 75.4, 61.0, 44.1, 32.6, 32.3, 29.8, 29.7, 29.5, 29.4, 23.1 and 14.5; $\mathrm{m} / \mathrm{z}$ (low resolution, ES) $307.2\left(52 \%, \mathrm{M}^{+}+\mathrm{CH}_{4}{ }^{+} \mathrm{CN}\right), 304.1(100 \%)$ and $266.2\left(57 \%, \mathrm{M}^{+}+\mathrm{H}\right) ; \mathrm{m} / \mathrm{z}$ (accurate mass, ES) Found: $\mathrm{M}^{+}+\mathrm{H}, 266.2113 . \mathrm{C}_{16} \mathrm{H}_{28} \mathrm{NO}_{2}$ requires $\mathrm{M}, 266.2120$. An enantiomeric excess of $93 \%$ was 
determined by chiral HPLC using a Daicel Chemical Industries Ltd. Chiralpak AS $(250 \times 4.6 \mathrm{~mm})$ HPLC column, eluting with $10 \%$ IPA in hexane at a rate of $1 \mathrm{ml} / \mathrm{min}$ with retention times of 21 and 36 minutes.

(1'E,4S)-4-Benzyloxymethyl-4-dec-1'-enyloxazolidin-2-one 12e

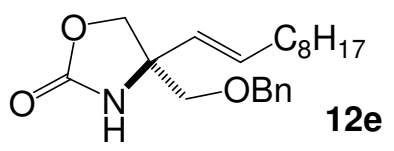

Colourless oil; $R_{\mathrm{F}} 0.30$ (60\% $\mathrm{Et}_{2} \mathrm{O}$ in petrol); $[\alpha]_{\mathrm{D}}{ }^{24}-45.48$ (c. 0.73 in $\mathrm{CHCl}_{3}$ ); (Found: C, 72.85; $\mathrm{H}, 9.0$; N, 4.3; $\mathrm{C}_{21} \mathrm{H}_{31} \mathrm{NO}_{3}$ requires: $\left.\mathrm{C}, 73.01 ; \mathrm{H}, 9.04 ; \mathrm{N}, 4.05 \%\right) ; v_{\max } / \mathrm{cm}^{-1}\left(\mathrm{CHCl}_{3}\right.$ solution) 3270, 1754, 1454, 1097 and 1044; $\delta_{\mathrm{H}}\left(300 \mathrm{MHz}, \mathrm{CDCl}_{3}\right)$ 7.40-7.25 $(5 \mathrm{H}, \mathrm{m}, \mathrm{Ph}), 5.76\left(1 \mathrm{H}, \mathrm{dt}, J 15.9\right.$ and 6.7, $\left.\mathrm{H}-2{ }^{\prime}\right), 5.68(1 \mathrm{H}, \mathrm{s}, \mathrm{NH}), 5.48(1 \mathrm{H}, \mathrm{d}, J$ 15.9, H-1'), $4.56\left(2 \mathrm{H}, \mathrm{s}, \mathrm{CH}_{2} \mathrm{Ph}\right), 4.30\left(1 \mathrm{H}, \mathrm{d}, \mathrm{J} 8.5, \mathrm{H}-5_{\mathrm{A}}\right), 4.07\left(1 \mathrm{H}, \mathrm{d}, J\right.$ 8.5, H-5 $\left.{ }_{\mathrm{B}}\right), 3.45\left(2 \mathrm{H}, \mathrm{s}, \mathrm{CH}_{2} \mathrm{OBn}\right), 2.04(2 \mathrm{H}$, apparent q, J 6.7, $\left.\mathrm{H}-3^{\prime}\right), 1.42-1.18\left(12 \mathrm{H}, \mathrm{m}, \mathrm{CH}_{2} \times 6\right)$ and $0.88\left(3 \mathrm{H}, \mathrm{t}, J 6.4, \mathrm{H}-10^{\prime}\right) ; \delta_{\mathrm{C}}\left(75 \mathrm{MHz}, \mathrm{CDCl}_{3}\right) 159.5$, 137.8, 133.4, 128.9, 128.4, 128.1, 125.9, 74.4, 74.0, 73.6, 61.5, 32.7, 32.3, 29.8, 29.7, 29.5, 29.3, 23.1 and 14.5; $\mathrm{m} / \mathrm{z}$ (low resolution, ES) $387.1\left(77 \%, \mathrm{M}^{+}+\mathrm{CH}_{4}{ }^{+} \mathrm{CN}\right), 363.2\left(100 \%, \mathrm{M}^{+}+\mathrm{NH}_{4}{ }^{+}\right)$and $346.1\left(17 \%, \mathrm{M}^{+}+\mathrm{H}\right)$. An enantiomeric excess of $93 \%$ was determined by chiral HPLC using a Daicel Chemical Industries Ltd. Chiralpak OJ $(250 \times 4.6 \mathrm{~mm}) \mathrm{HPLC}$ column, eluting with $5 \%$ IPA in hexane at a rate of $1 \mathrm{ml} / \mathrm{min}$ with retention times of 13 and 21 minutes.

(1'E,4R)-4-Dec-1'-enyl-4-(2''-hydroxyethyl)oxazolidin-2-one $12 f$ and (1'E,4R)-4-dec-1'-enyl-4-hydroxymethyl$(1,3)$-oxazinan-2-one 12f'<smiles>CCCC=CC1(CCO)COC(=O)N1</smiles>

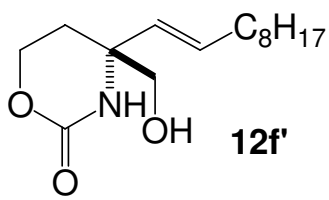

12f, colourless oil; $R_{\mathrm{F}} 0.33$ (80\% EtOAc in petrol); $[\alpha]_{\mathrm{D}}^{24}-28.03$ (c. 0.79 in $\left.\mathrm{CHCl}_{3}\right) ; v_{\max } / \mathrm{cm}^{-1}\left(\mathrm{CHCl}_{3}\right.$ solution) 3305, 1748, 1467, 1394, 1276, 1037 and 972; $\delta_{\mathrm{H}}\left(300 \mathrm{MHz}, \mathrm{CDCl}_{3}\right) 6.47(1 \mathrm{H}, \mathrm{s}, \mathrm{NH}), 5.68(1 \mathrm{H}, \mathrm{dt}, J 15.4$ and 6.9, H-2'), $5.35\left(1 \mathrm{H}, \mathrm{d}, J\right.$ 15.6, H-1'), $4.12\left(1 \mathrm{H}, \mathrm{d}, J\right.$ 8.2, H-5 $\left.{ }_{\mathrm{A}}\right), 4.07$ (1H, d, J 8.5, H-5 $\mathrm{B}_{\mathrm{B}}$, $3.71\left(2 \mathrm{H}, \mathrm{m}, \mathrm{H}-2{ }^{\prime \prime}\right), 2.69$ (1H, s, $\mathrm{OH})$, 2.08-1.86 (3H, m, H-3' and $\left.\mathrm{H}-1{ }_{\mathrm{A}}\right), 1.72\left(1 \mathrm{H}, \mathrm{dt}, J 14.1\right.$ and $\left.4.1, \mathrm{H}-1{ }_{\mathrm{B}}\right), 1.36-1.12\left(12 \mathrm{H}, \mathrm{m}, \mathrm{CH}_{2} \times 6\right)$ and 0.81 $\left(3 \mathrm{H}, \mathrm{t}, J\right.$ 6.9, $\left.\mathrm{H}-10^{\prime}\right) ; \delta_{\mathrm{C}}\left(75 \mathrm{MHz}, \mathrm{CDCl}_{3}\right)$ 160.0, 132.4, 130.6, 76.7, 61.4, 59.1, 41.0, 32.6, 32.3, 29.8, 29.7, 29.6, 29.5, 23.1 and 14.5; m/z (low resolution, ES) $270.2\left(90 \%, \mathrm{M}^{+}+\mathrm{H}\right) ; \mathrm{m} / \mathrm{z}$ (accurate mass, ES) Found: $\mathrm{M}^{+}+\mathrm{H}, 270.2067$. $\mathrm{C}_{15} \mathrm{H}_{28} \mathrm{NO}_{3}$ requires $\mathrm{M}, 270.2069$. An enantiomeric excess of $95 \%$ was determined by chiral HPLC using a Daicel Chemical Industries Ltd. Chiralpak OD-H $(250 \times 4.6 \mathrm{~mm})$ HPLC column, eluting with 10\% IPA in hexane at a rate of $1 \mathrm{ml} / \mathrm{min}$ with retention times of 10 and 14 minutes.

12f', colourless oil; $R_{\mathrm{F}} 0.12$ (80\% EtOAc in petrol); $[\alpha]_{\mathrm{D}}^{24}+20.03$ (c. 0.28 in $\mathrm{CHCl}_{3}$ ); (Found: C, 66.7; $\mathrm{H}, 10.25 ; \mathrm{N}$, 5.0; $\mathrm{C}_{15} \mathrm{H}_{27} \mathrm{NO}_{3}$ requires: $\left.\mathrm{C}, 66.88 ; \mathrm{H}, 10.10 ; \mathrm{N}, 5.20 \%\right) ; v_{\max } / \mathrm{cm}^{-1}\left(\mathrm{CHCl}_{3}\right.$ solution) $3271,1698,1478,1427,1315$, 1112 and 1079; $\delta_{\mathrm{H}}\left(300 \mathrm{MHz}, \mathrm{CDCl}_{3}\right) 6.78(1 \mathrm{H}, \mathrm{s}, \mathrm{NH}), 5.70\left(1 \mathrm{H}, \mathrm{dt}, J 15.4\right.$ and 6.7, H-2'), $5.22\left(1 \mathrm{H}, \mathrm{d}, J 15.4, \mathrm{H}-1^{\prime}\right)$, $4.14(2 \mathrm{H}, \mathrm{m}, \mathrm{H}-6), 3.52\left(1 \mathrm{H}, \mathrm{d}, J 11.5, \mathrm{CH}_{A} \mathrm{H}_{\mathrm{B}} \mathrm{OH}\right), 3.40\left(1 \mathrm{H}, \mathrm{d}, J 11.5, \mathrm{CH}_{\mathrm{A}} \mathrm{H}_{B} \mathrm{OH}\right), 2.60(1 \mathrm{H}, \mathrm{brs}, \mathrm{OH}), 2.02(3 \mathrm{H}, \mathrm{m}$, $\mathrm{H}-3^{\prime}$ and $\left.\mathrm{H}-5_{\mathrm{A}}\right), 1.55\left(1 \mathrm{H}\right.$, brd, J 13.8, $\left.\mathrm{H}-5_{\mathrm{B}}\right), 1.36-1.13\left(12 \mathrm{H}, \mathrm{m}, \mathrm{CH}_{2} \times 6\right)$ and $0.81\left(3 \mathrm{H}, \mathrm{t}, J 6.7, \mathrm{H}-10^{\prime}\right) ; \delta_{\mathrm{C}}(75 \mathrm{MHz}$, $\mathrm{CDCl}_{3}$ ) 155.7, 135.3, 130.8, 68.9, 63.7, 59.9, 32.7, 32.3, 29.8, 29.7, 29.6, 29.6, 28.5, 23.1 and 14.5; $\mathrm{m} / \mathrm{z}$ (low resolution, ES) $561.1\left(20 \%, 2 \mathrm{M}^{+}+{ }^{23} \mathrm{Na}\right), 539.2\left(100 \%, 2 \mathrm{M}^{+}+\mathrm{H}\right), 311.0\left(52 \%, \mathrm{M}^{+}+\mathrm{CH}_{4}{ }^{+} \mathrm{CN}\right)$ and $270.0\left(60 \%, \mathrm{M}^{+}+\mathrm{H}\right)$. 
An enantiomeric excess of 95\% was determined by chiral HPLC using a Daicel Chemical Industries Ltd. Chiralpak OD-H $(250 \times 4.6 \mathrm{~mm}) \mathrm{HPLC}$ column, eluting with $10 \%$ IPA in hexane at a rate of $1 \mathrm{ml} / \mathrm{min}$ with retention times of 9 and 18 minutes.

\section{(4R,1'E)-4-Methyl-4-styryloxazolidin-2-one 12g}<smiles>C[C@]1(/C=C/[PbH])COC(=O)N1</smiles>

Colourless solid, $\mathrm{mp} 76.7-77.5^{\circ} \mathrm{C} ; R_{\mathrm{F}} 0.27$ (80\% $\mathrm{Et}_{2} \mathrm{O}$ in petrol); $[\alpha]_{\mathrm{D}}{ }^{24}-24.87$ (c. 1.34 in $\mathrm{CHCl}_{3}$ ); (Found: C, 71.0; $\mathrm{H}, 6.65 ; \mathrm{N}, 6.7 ; \mathrm{C}_{12} \mathrm{H}_{13} \mathrm{NO}_{2}$ requires: $\left.\mathrm{C}, 70.92 ; \mathrm{H}, 6.45 ; \mathrm{N}, 6.89 \%\right) ; v_{\max } / \mathrm{cm}^{-1}\left(\mathrm{CHCl}_{3}\right.$ solution) 3271, 1748, 1391, 1288, 1038 and 969; $\delta_{\mathrm{H}}\left(300 \mathrm{MHz}, \mathrm{CDCl}_{3}\right) 7.40-7.23(5 \mathrm{H}, \mathrm{m}, \mathrm{Ph}), 6.61(1 \mathrm{H}, \mathrm{d}, J 16.1, \mathrm{PhCHCH}), 6.27(1 \mathrm{H}, \mathrm{s}, \mathrm{NH})$, $6.22(1 \mathrm{H}, \mathrm{d}, J$ 16.1, PhCHCH $), 4.25(1 \mathrm{H}, \mathrm{d}, J$ 8.4, H-5 $), 4.17(1 \mathrm{H}, \mathrm{d}, J$ 8.4, H-5 $)$ and $1.58(3 \mathrm{H}, \mathrm{s}, \mathrm{Me}) ; \delta_{\mathrm{C}}(75 \mathrm{MHz}$, $\left.\mathrm{CDCl}_{3}\right)$ 159.6, 136.1, 131.6, 130.4, 129.1, 128.7, 127.1, 76.9, 59.0 and 26.0; m/z (low resolution, ES) 244.9 (100\%, $\mathrm{M}^{+}+\mathrm{CH}_{4}{ }^{+} \mathrm{CN}$ ). An enantiomeric excess of $93 \%$ was determined by chiral HPLC using a Daicel Chemical Industries Ltd. Chiralpak OJ $(250 \times 4.6 \mathrm{~mm})$ HPLC column, eluting with $5 \%$ IPA in hexane at a rate of $1 \mathrm{ml} / \mathrm{min}$ with retention times of 51 and 81 minutes.

\section{$\left(4 S, 1^{\prime} E\right)-4-B e n z y l o x y m e t h y l-4-s t y r y l o x a z o l i d i n-2-o n e ~ 12 h$}

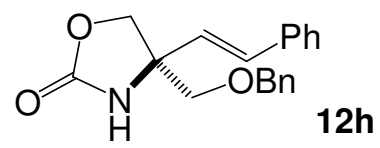

Colourless oil; $R_{\mathrm{F}} 0.39\left(80 \% \mathrm{Et}_{2} \mathrm{O}\right.$ in petrol); $[\alpha]_{\mathrm{D}}^{23}-58.24$ (c. 1.48 in $\mathrm{CHCl}_{3}$ ); (Found: C, 73.5; $\mathrm{H}, 6.4 ; \mathrm{N}, 4.65$; $\mathrm{C}_{19} \mathrm{H}_{19} \mathrm{NO}_{3}$ requires: $\left.\mathrm{C}, 73.77 ; \mathrm{H}, 6.19 ; \mathrm{N}, 4.53 \%\right) ; v_{\max } / \mathrm{cm}^{-1}$ ( $\mathrm{CHCl}_{3}$ solution) 3271, 1750, 1496, 1452, 1391, 1096 and 1043; $\delta_{\mathrm{H}}\left(300 \mathrm{MHz}, \mathrm{CDCl}_{3}\right) 7.40-7.22(10 \mathrm{H}, \mathrm{m}, \mathrm{Ph}), 6.68(1 \mathrm{H}, \mathrm{d}, J 16.1, \mathrm{PhCHCH}), 6.27(1 \mathrm{H}, \mathrm{s}, \mathrm{NH}), 6.21(1 \mathrm{H}$, $\mathrm{d}, J$ 16.1, $\mathrm{PhCHCH}), 4.57\left(2 \mathrm{H}, \mathrm{s}, \mathrm{CH}_{2} \mathrm{Ph}\right), 4.40\left(1 \mathrm{H}, \mathrm{d}, J\right.$ 8.5, H-5 $5_{\mathrm{A}}, 4.17\left(1 \mathrm{H}, \mathrm{d}, J 8.5, \mathrm{H}-5_{\mathrm{B}}\right)$ and $3.56(2 \mathrm{H}, \mathrm{s}$, $\left.\mathrm{CH}_{2} \mathrm{OBn}\right) ; \delta_{\mathrm{C}}\left(75 \mathrm{MHz}, \mathrm{CDCl}_{3}\right) 159.7,137.7,136.1,131.7,129.1,129.0,128.7,128.5,128.3,128.2,127.1,74.3$, 74.0, 73.5 and 62.0; $\mathrm{m} / \mathrm{z}$ (low resolution, ES) $350.9\left(100 \%, \mathrm{M}^{+}+\mathrm{CH}_{4}{ }^{+} \mathrm{CN}\right), 326.9\left(42 \%, \mathrm{M}^{+}+\mathrm{NH}_{4}{ }^{+}\right)$and $309.9(22 \%$, $\mathrm{M}^{+}+\mathrm{H}$ ). An enantiomeric excess of $95 \%$ was determined by chiral HPLC using a Daicel Chemical Industries Ltd. Chiralpak OJ $(250 \times 4.6 \mathrm{~mm})$ HPLC column, eluting with $20 \%$ IPA in hexane at a rate of $1 \mathrm{ml} / \mathrm{min}$ with retention times of 53 and 68 minutes.

\section{(4R,1'E)-4-Methyl-4-(3'-phenylpropenyl)oxazolidin-2-one 12i}

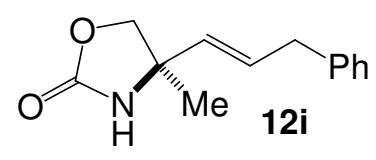

Colourless oil; $R_{\mathrm{F}} 0.42$ (80\% $\mathrm{Et}_{2} \mathrm{O}$ in petrol); $[\alpha]_{\mathrm{D}}{ }^{24}-20.74$ (c. 0.68 in $\mathrm{CHCl}_{3}$ ); (Found: C, 71.6; $\mathrm{H}, 7.2 ; \mathrm{N}, 6.7$; $\mathrm{C}_{13} \mathrm{H}_{15} \mathrm{NO}_{2}$ requires: $\left.\mathrm{C}, 71.87 ; \mathrm{H}, 6.96 ; \mathrm{N}, 6.45 \%\right) ; v_{\max } / \mathrm{cm}^{-1}$ ( $\mathrm{CHCl}_{3}$ solution) $3270,1749,1495,1476,1453,1391$, 1287 and 1038; $\delta_{\mathrm{H}}\left(300 \mathrm{MHz}, \mathrm{CDCl}_{3}\right) 7.35-7.10(5 \mathrm{H}, \mathrm{m}, \mathrm{Ph}), 6.34(1 \mathrm{H}, \mathrm{s}, \mathrm{NH}), 5.86(1 \mathrm{H}, \mathrm{dt}, J 15.6$ and 6.7, H-2'), $5.56\left(1 \mathrm{H}, \mathrm{d}, J 15.6, \mathrm{H}-1^{\prime}\right), 4.14\left(1 \mathrm{H}, \mathrm{d}, J 8.5, \mathrm{H}-5_{\mathrm{A}}\right), 4.08\left(1 \mathrm{H}, \mathrm{d}, J\right.$ J $\left.8.2, \mathrm{H}-5_{\mathrm{B}}\right), 3.38(2 \mathrm{H}, \mathrm{d}, J$ 6.7, H-3') and $1.44(3 \mathrm{H}$, $\mathrm{s}, \mathrm{Me}$ ); $\delta_{\mathrm{C}}\left(75 \mathrm{MHz}, \mathrm{CDCl}_{3}\right)$ 159.7, 139.7, 133.8, 130.4, 129.0, 129.0, 126.8, 76.9, 58.7, 38.8 and 25.9; m/z (low resolution, ES) $258.9\left(100 \%, \mathrm{M}^{+}+\mathrm{CH}_{4}{ }^{+} \mathrm{CN}\right), 234.9\left(38 \%, \mathrm{M}^{+}+\mathrm{NH}_{4}{ }^{+}\right)$and $217.9\left(5 \%, \mathrm{M}^{+}+\mathrm{H}\right)$. An enantiomeric excess 
of $95 \%$ was determined by chiral HPLC using a Daicel Chemical Industries Ltd. Chiralpak OJ $(250 \times 4.6 \mathrm{~mm}) \mathrm{HPLC}$ column, eluting with $10 \%$ IPA in hexane at a rate of $1 \mathrm{ml} / \mathrm{min}$ with retention times of 19 and 43 minutes.

\section{(4S,1'E)-4-Benzyloxymethyl-4-(3'-phenylpropenyl)oxazolidin-2-one 12j}

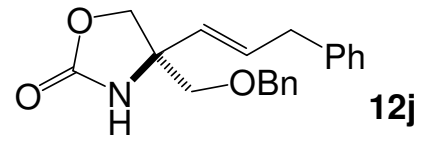

Colourless oil; $R_{\mathrm{F}} 0.48$ (80\% $\mathrm{Et}_{2} \mathrm{O}$ in petrol); $[\alpha]_{\mathrm{D}}{ }^{24}-60.39$ (c. 0.52 in $\left.\mathrm{CHCl}_{3}\right) ; v_{\max } / \mathrm{cm}^{-1}\left(\mathrm{CHCl}_{3}\right.$ solution) 3270 , 1748, 1495, 1472, 1453, 1393, 1266 and 1044; $\delta_{\mathrm{H}}\left(300 \mathrm{MHz}, \mathrm{CDCl}_{3}\right)$ 7.38-7.10 (10H, m, Ph), $6.07(1 \mathrm{H}, \mathrm{s}, \mathrm{NH})$, $5.92\left(1 \mathrm{H}, \mathrm{dt}, J 15.6\right.$ and 6.7, $\left.\mathrm{H}-2{ }^{\prime}\right), 5.53\left(1 \mathrm{H}, \mathrm{dt}, J 15.6\right.$ and 1.3, $\left.\mathrm{H}-1^{\prime}\right), 4.53\left(2 \mathrm{H}, \mathrm{s}, \mathrm{OCH} \mathrm{H}_{2} \mathrm{Ph}\right), 4.30(1 \mathrm{H}, \mathrm{d}, J$ 8.4, H$\left.5_{\mathrm{A}}\right), 4.07\left(1 \mathrm{H}, \mathrm{d}, J\right.$ 8.4, H-5 $\left.\left.5_{\mathrm{B}}\right), 3.45\left(2 \mathrm{H}, \mathrm{s}, \mathrm{CH} \mathrm{H}_{2} \mathrm{OBn}\right), 3.38(2 \mathrm{H}, \mathrm{d}, J 6.7, \mathrm{H}-3)^{\prime}\right) ; \delta_{\mathrm{C}}\left(75 \mathrm{MHz}, \mathrm{CDCl}_{3}\right) 159.6,139.6$, 137.8, 131.8, 130.5, 129.0, 129.0, 129.0, 128.4, 128.1, 126.8, 74.3, 74.0, 73.4, 61.6 and 38.9; $\mathrm{m} / \mathrm{z}$ (low resolution, ES) $364.7\left(74 \%, \mathrm{M}^{+}+\mathrm{CH}_{4}{ }^{+} \mathrm{CN}\right), 345.7\left(38 \%, \mathrm{M}^{+}+{ }^{23} \mathrm{Na}\right)$ and $323.8\left(100 \%, \mathrm{M}^{+}+\mathrm{H}\right) ; \mathrm{m} / \mathrm{z}$ (accurate mass, ES) Found: $\mathrm{M}^{+}+\mathrm{H}, 324.1605 . \mathrm{C}_{20} \mathrm{H}_{22} \mathrm{NO}_{3}$ requires $\mathrm{M}, 324.1600$. An enantiomeric excess of $92 \%$ was determined by chiral HPLC using a Daicel Chemical Industries Ltd. Chiralpak AS $(250 \times 4.6 \mathrm{~mm})$ HPLC column, eluting with $20 \%$ IPA in hexane at a rate of $1 \mathrm{ml} / \mathrm{min}$ with retention times of 95 and 106 minutes.

\section{(2R,3E)-2-Amino-2-methyldodec-3-enoic acid 13}<smiles>CC(N)(C=CC[18F])C(=O)O</smiles>

13

Water $(8.1 \mu \mathrm{l}, 0.45 \mathrm{mmol}$ ) was added to a stirred suspension of $\alpha, \alpha$-disubstituted amino acid $4 \mathrm{a}$ (89 $\mathrm{mg}, 0.15$ $\mathrm{mmol}$ ) and potassium tert-butoxide $(173 \mathrm{mg}, 1.55 \mathrm{mmol})$ in 1,4-dioxane $(2 \mathrm{ml})$ under nitrogen at room temperature. The reaction mixture was refluxed for 17 hours. The reaction mixture was cooled to room temperature, quenched with water $(2 \mathrm{ml})$ and the solution was neutralised with Amberlite IRC-50 $\left(\mathrm{H}^{+}\right.$form). The solution was filtered and the resin washed with methanol $(10 \mathrm{ml})$. The filtrate was evaporated under reduced pressure to give a crude product, which was purified by flash chromatography, eluting with 95:5 chloroform-methanol, to give (-)-8phenylmenthol (29 mg, $82 \%$ ) as a colourless oil and then 10:3:1 chloroform-methanol-water (the lower phase of this mixture was used), to give amino acid 13 (30 mg, 88\%) as a colourless powder, $\mathrm{mp} 216.3-218.9{ }^{\circ} \mathrm{C} ; R_{\mathrm{F}} 0.30$ (10:3:1 $\mathrm{CHCl}_{3}-\mathrm{MeOH}-\mathrm{H}_{2} \mathrm{O}$ \{lower phase\}); $[\alpha]_{\mathrm{D}}{ }^{24}-1.17$ (c. 0.98 in $\mathrm{MeOH}$ ); $v_{\max } / \mathrm{cm}^{-1}$ (diamond transition cell) 3308-2108, 1655, 1611, 1496, 1455, 1389 and 1358; $\delta_{\mathrm{H}}\left(300 \mathrm{MHz}, \mathrm{CD}_{3} \mathrm{OD}\right) 5.72(1 \mathrm{H}, \mathrm{dt}, J 15.9$ and 6.4, H-4), 5.60 $(1 \mathrm{H}, \mathrm{d}, J$ 15.9, $\mathrm{H}-3), 2.00(2 \mathrm{H}, \mathrm{dt}, J 6.9$ and 6.7, $\mathrm{H}-5), 1.45(3 \mathrm{H}, \mathrm{s}, \mathrm{Me}), 1.40-1.13\left(12 \mathrm{H}, \mathrm{m}, \mathrm{CH}_{2} \times 6\right)$ and $0.81(3 \mathrm{H}, \mathrm{t}$, J 6.9, $\mathrm{H}-12) ; \delta_{\mathrm{C}}\left(75 \mathrm{MHz}, \mathrm{CD}_{3} \mathrm{OD}\right) 175.9,133.6,130.7,62.8,33.9,33.4,30.9,30.7,30.6,30.4,24.1,23.6$ and 14.8; $\mathrm{m} / \mathrm{z}$ (low resolution, ES) $269.1\left(100 \%, \mathrm{M}^{+}+\mathrm{CH}_{4}{ }^{+} \mathrm{CN}\right)$ and $228.1\left(100 \%, \mathrm{M}^{+}+\mathrm{H}\right) ; \mathrm{m} / \mathrm{z}$ (accurate mass, ES) Found: $\mathrm{M}^{+}+\mathrm{H}$, 228.1955. $\mathrm{C}_{13} \mathrm{H}_{26} \mathrm{NO}_{2}$ requires $\mathrm{M}, 228.1964$. 
As part of a subsequent application to natural product synthesis, the following alkylation was undertaken, and the crystalline derivative $\mathbf{1 4}$ then obtained. Full details of the synthesis of $\mathbf{1 4}$ along with the X-ray crystal data will be disclosed in a future publication.

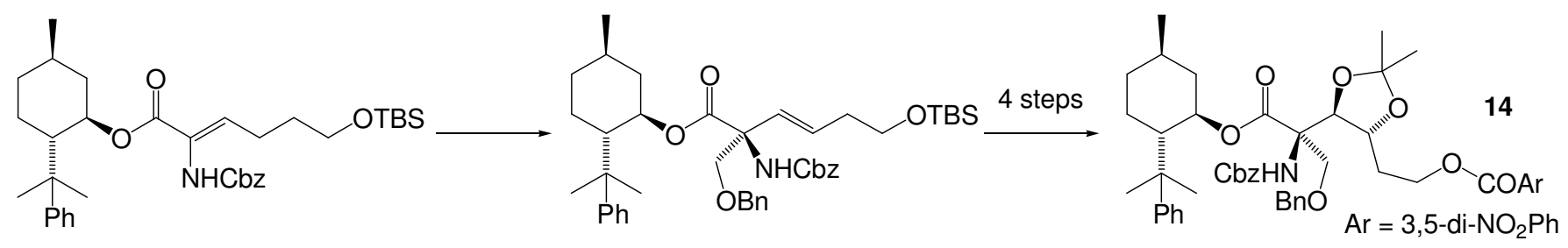

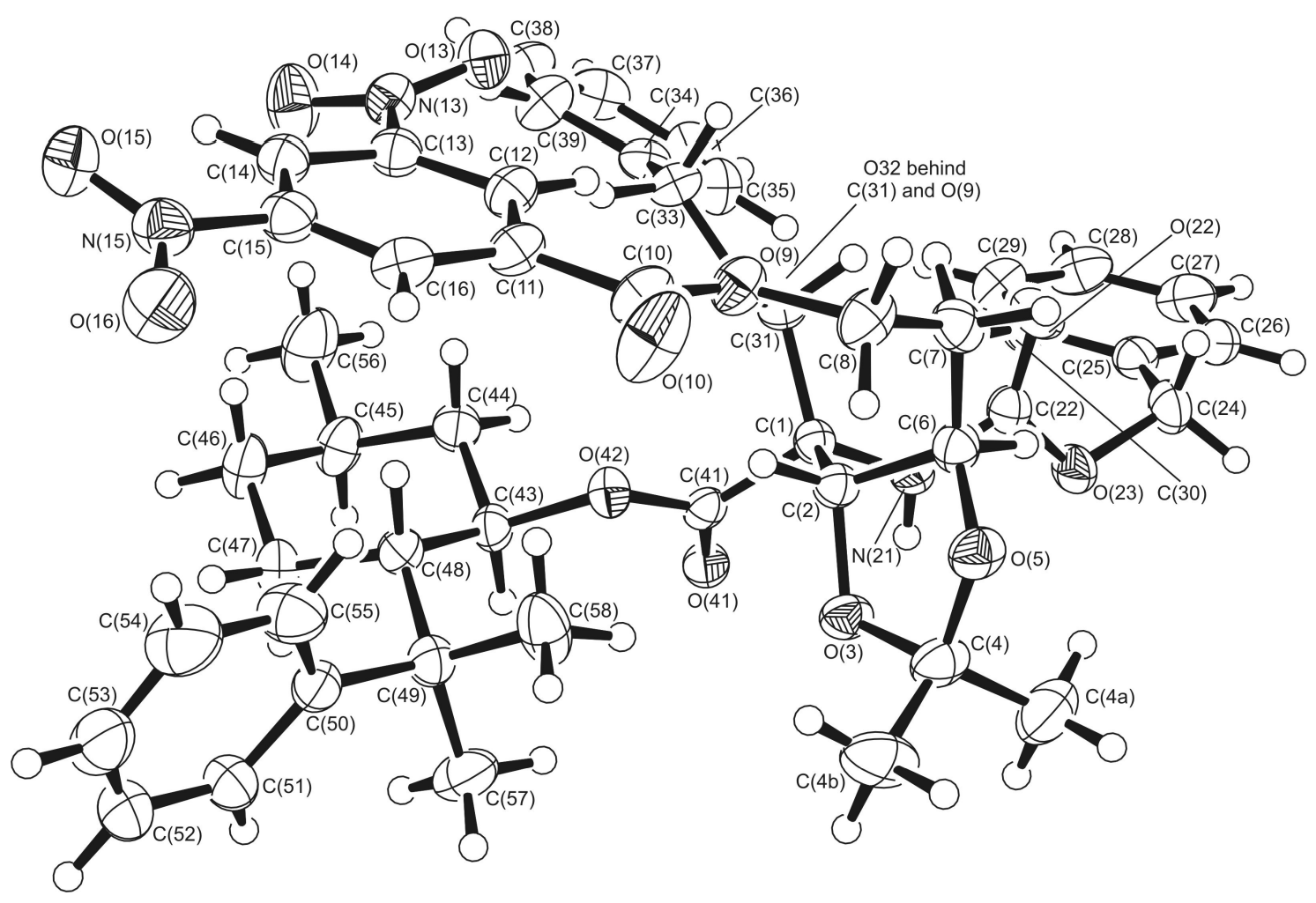

